\title{
THE UNDECIDABILITY OF THE INFINITE RIBBON PROBLEM: IMPLICATIONS FOR COMPUTING BY SELF-ASSEMBLY*
}

\author{
LEONARD ADLEMAN ${ }^{\dagger}$, JARKKO KARI $^{\ddagger}$, LILA KARI $^{\S}$, DUSTIN REISHUS $^{\dagger}$, AND \\ PETR SOSIK
}

\begin{abstract}
Self-assembly, the process by which objects autonomously come together to form complex structures, is omnipresent in the physical world. Recent experiments in self-assembly demonstrate its potential for the parallel creation of a large number of nanostructures, including possibly computers. A systematic study of self-assembly as a mathematical process has been initiated by L. Adleman and E. Winfree. The individual components are modeled as square tiles on the infinite two-dimensional plane. Each side of a tile is covered by a specific "glue," and two adjacent tiles will stick iff they have matching glues on their abutting edges. Tiles that stick to each other may form various two-dimensional "structures" such as squares and rectangles, or may cover the entire plane. In this paper we focus on a special type of structure, called a ribbon: a non-self-crossing rectilinear sequence of tiles on the plane, in which successive tiles are adjacent along an edge and abutting edges of consecutive tiles have matching glues. We prove that it is undecidable whether an arbitrary finite set of tiles with glues (infinite supply of each tile type available) can be used to assemble an infinite ribbon. While the problem can be proved undecidable using existing techniques if the ribbon is required to start with a given "seed" tile, our result settles the "unseeded" case, an open problem formerly known as the "unlimited infinite snake problem." The proof is based on a construction, due to R. Robinson, of a special set of tiles that allow only aperiodic tilings of the plane. This construction is used to create a special set of directed tiles (tiles with arrows painted on the top) with the "strong plane-filling property" - a variation of the "plane-filling property" previously defined by J. Kari. A construction of "sandwich" tiles is then used in conjunction with this special tile set, to reduce the well-known undecidable tiling problem to the problem of the existence of an infinite directed zipper (a special kind of ribbon). A "motif" construction is then introduced that allows one tile system to simulate another by using geometry to represent glues. Using motifs, the infinite directed zipper problem is reduced to the infinite ribbon problem, proving the latter undecidable. An immediate consequence of our result is the undecidability of the existence of arbitrarily large structures self-assembled using tiles from a given tile set.
\end{abstract}

Key words. DNA computing, molecular computing, self-assembly, tiling, undecidability

AMS subject classifications. 68Q80, 37B15, 03D35, 92B05

DOI. $10.1137 / 080723971$

* Received by the editors May 12, 2008; accepted for publication (in revised form) December 12, 2008; published electronically March 20, 2009. This paper is based on "On the decidability of selfassembly of infinite ribbons," by L. Adleman, J. Kari, L. Kari, and D. Reishus, which appeared in Proceedings of the IEEE Symposium on Foundations of Computer Science (FOCS), 2002, pp. 530-537. (c) 2002 IEEE.

http://www.siam.org/journals/sicomp/38-6/72397.html

$\dagger$ Department of Computer Science, University of Southern California, 3710 McClintock Ave., RTH 501, Los Angeles, CA 90089-2905 (adleman@usc.edu, reishus@usc.edu). The first author's research was supported by NASA/JPL, NSF, ONR, and DARPA grants.

${ }^{\ddagger}$ Department of Mathematics, University of Turku, Turku FI-20014, Finland (jkari@utu.fi). This author's research was supported by NSF grant CCR 97-33101 and Academy of Finland grant 211967.

$\S$ Department of Computer Science, University of Western Ontario, London, ON, N6A 5B7, Canada (lila@csd.uwo.ca). This author's research was supported by a Canada Research Chair Award and a discovery grant of the Natural Sciences and Engineering Research Council of Canada.

『Institute of Computer Science, Silesian University in Opava, 74601 Opava, Czech Republic, and Department of Artificial Intelligence, Polytechnical University of Madrid, 28660 Madrid, Spain (petr.sosik@fpf.slu.cz). This author's research was supported by grant 201/06/0567 of the Czech Science Foundation and the Ramón y Cajal Program of the Ministry of Science and Technology of Spain. 
1. Introduction. Self-assembly, the process by which objects autonomously come together to form complex structures, is omnipresent in the physical world. Atoms bind to each other by chemical bonds to form molecules, molecules may form crystals or macromolecules, and cells interact to form biological organisms. Recently it has been suggested that complex self-assembly processes will ultimately be used in circuit fabrication, nanorobotics, DNA computation, and amorphous computing. Indeed, in electronics, engineering, medicine, material science, manufacturing, and other disciplines, there is a continuous drive toward miniaturization. Unfortunately, current "top-down" techniques such as lithography may not be capable of efficiently creating structures with features the size of molecules or atoms. Self-assembly provides a "bottom-up" approach by which such fine structures might be created.

Experimental research on self-assembly includes simulation of one-dimensional cellular automata by using macroscopic plastic tiles that assemble at an oil/water interface [38], studies of self-directed growth of hybrid organic molecules on a silicon substrate [31], self-assembly of regular lipid hollow icosahedra [14], self-assembly of patterns of lead on a copper surface as templates for fabricating nanostructures [35], formation of electrical networks by self-assembly of small plastic and metal objects [22], [49], self-assembly of molecular machines [20], the construction by self-assembly of a molecular-thickness transistor [43], and DNA nanomachines made by self-assembly (e.g., molecular switches [30], tweezers [53], [33], walkers [44], autonomous DNA motors [47], [9], [23]), as well as algorithmic self-assembled Sierpinski triangles [41], self-assembled cloneable DNA octahedra [45], stiff self-assembled tetrahedra for threedimensional electronic circuits [21], and DNA origami [40].

Investigations into DNA computing [2] and amorphous computing [1] have pointed out a strong connection between self-assembly and computation. While [52], [29], [36], [50] have shown the potential of DNA self-assembly for computation, [51] has experimentally demonstrated the self-assembly of periodic two-dimensional arrays from DNA "tiles," in which "binding" is regulated by the "sticky" ends of the DNA tiles. Reference [32] has demonstrated the execution of logical operations (cumulative XOR) by self-assembly of DNA triple cross-over molecules and [10] has demonstrated the potential for programmable self-assembled computing devices.

A systematic study of self-assembly as a computational process has been initiated in [3]. The individual components are therein modeled as square tiles on the infinite two-dimensional plane. The tiles cannot be rotated. Each side of a tile is covered by a specific "glue," and two adjacent tiles will stick iff they have matching glues on their abutting edges. As such, tiles have been previously studied in the context of classic questions about tilings of the plane [48]. The tiling problem, for example (proved undecidable in [11], [37]), asks whether a given set of tiles (supply of each tile type unlimited) can be used to correctly tile the entire plane. However, the new requirements of experimental self-assembly of tiles into required nanoscale shapes pose new types of questions. What is the minimal number of tiles required for the selfassembly of a desired shape [5], [19], [13], [46], [25], [39]? What is the running time of such a self-assembly [5], [4]? Do the results still hold if one generalizes the model by making the "sticking" reversible, or by defining different bond strengths and requiring more than one bond for sticking to occur [42], [4], [6], [5]? Do reversible self-assemblies achieve equilibrium [6]? What is the optimal initial concentration of tile types that guarantees the fastest assembly of a required shape [5]? Can a self-assembled shape recover from the loss of arbitrary many tiles [12]? What are possible computational primitives for algorithmic self-assembly [8]?

One of the interesting problems of self-assembly is whether or not a given set of 
tiles allows uncontrollable growth, that is, whether arbitrarily large structures can be produced. It turns out that this question is equivalent to asking whether or not, given a set of tiles, there exists an infinite ribbon that can be formed with tiles from this set. A ribbon is a non-self-crossing sequence of tiles on the plane, in which successive tiles are adjacent along an edge and abutting edges of consecutive tiles have matching glues. If a given "seed" tile is specified, the problem of existence of an infinite ribbon starting at that tile has been proved undecidable [16], [15], [17]. However, when no such seed is specified, existing proof techniques failed to produce an answer and the problem was declared open in [17]. The unseeded problem is also relevant given that, in some physical simulations of self-assembly [38], the growth mechanism was deemed incompatible with computations that use a chosen input. (More recently, in [18], successful experimental "seeded" self-assembly of fixed-length Sierpinski-patterned ribbons was reported. The assemblies, which use DNA tiles, start their growth from DNA "origami seeds" - special DNA tiles obtained by using the origami method [40].) Here we prove that the unseeded problem is also undecidable. This result settles an open problem known hitherto as the "unlimited infinite snake problem" [17]. Our results are placed within the framework of the "classical" tiling by self-assembly, in that our model does not take into account physical phenomena that may affect experimental DNA self-assembly such as concentrations of tiles, different glue strengths, and glue-matching errors.

The paper is organized as follows. Section 2 introduces the notions of tile, glue, tile system, sticking, ribbon, zipper, valid tiling of the plane, and directed tiles. In particular, a zipper is a ribbon with the additional requirement that even nonconsecutive tiles that touch must have matching glues at their abutting edges.

Section 3 starts with defining a directed tile system with the strong plane-filling property: in such a system, any infinite directed zipper is forced to follow a specific plane-filling self-similar path, and, moreover, an infinite directed zipper is always guaranteed to exist. The construction of a directed tile system with the above property uses a $3 \times 3$ block construction based on directed tiles defined in [27], [26], which, in turn, resemble tiles devised by Robinson in [37] to produce only aperiodic tilings of the plane. These tiles were augmented in [26], [27] with directions that forced any directed tiled path to form a self-similar plane-filling Hilbert curve that recursively fills arbitrarily large squares by filling each of their quadrants. The original construction used the condition that no glue mismatch be present in the $3 \times 3$ neighborhood of any tile on the path, to force the path to follow the desired curve. Here, the additional $3 \times 3$ block construction is needed to ensure that the weaker condition of no glue mismatch between any two tiles on the directed tiled path is enough to force its course.

Section 4 then proves the undecidability of the existence of an infinite directed zipper by reducing the tiling problem to it. The reduction is based on a construction of sandwich tiles, which have directed tiles from the directed tile system with the strong plane-filling property on top and undirected tiles from a given tile system on the bottom. The next result of the section proves the undecidability of the existence of an infinite ribbon by reducing the undecidable infinite directed zipper problem to it. The proof uses a "ribbon motif" construction that simulates each directed zipper tile by a ribbon of undirected tiles following its contours, and it uses geometry (bumps and dents) to simulate zipper-tile glues.

Sections 5 and 6 point to the implications of the undecidability of existence of infinite ribbons for the problem of self-assembly of arbitrarily large supertiles, and they summarize our results. 
2. Notation and definitions. A tile is an oriented unit square. The north, east, south, and west edges of the tile are each labeled with a symbol called a glue from a finite alphabet $X$. Tiles can be placed on the plane but not rotated. The positions of the tiles on the plane are indexed by $\mathbb{Z}^{2}$, the set of pairs of integers.

Formally, a tile $t$ is a quadruple $t=\left(t_{N}, t_{E}, t_{S}, t_{W}\right) \in X^{4}$, where $X$ is a finite set. The components $t_{N}, t_{E}, t_{S}, t_{W}$ will be called the glues on the north, east, south, and west edges of the tile, respectively. A tile system $T$ is a finite subset of $X^{4}$. For all tiles $t=\left(t_{N}, t_{E}, t_{S}, t_{W}\right)$ and $t^{\prime}=\left(t_{N}^{\prime}, t_{E}^{\prime}, t_{S}^{\prime}, t_{W}^{\prime}\right), t$ sticks on the north to $t^{\prime}$ iff $t_{N}=t_{S}^{\prime}$. Sticking on the east, south, and west is defined similarly.

The directions $\mathcal{D}=\{N, E, S, W\}$ are functions from $\mathbb{Z}^{2}$ to $\mathbb{Z}^{2}: N(x, y)=(x, y+1)$, $E(x, y)=(x+1, y), S(x, y)=(x, y-1), W(x, y)=(x-1, y)$. We say that the positions $(x, y)$ and $\left(x^{\prime}, y^{\prime}\right)$ are adjacent iff $\left(x^{\prime}, y^{\prime}\right) \in\{N(x, y), E(x, y), S(x, y), W(x, y)\}$. In addition, $(x, y)$ abuts $\left(x^{\prime}, y^{\prime}\right)$ on the north iff $\left(x^{\prime}, y^{\prime}\right)=N(x, y)$, and similarly for the other directions.

Given a tile system $T$, a $T$-tiling of the plane is a total function $f: \mathbb{Z}^{2} \longrightarrow T$. The tiling $f$ is valid at position $(x, y)$ iff $f(x, y)$ sticks on the north to $f(N(x, y))$, $f(x, y)$ sticks on the east to $f(E(x, y)), f(x, y)$ sticks on the south to $f(S(x, y))$, and $f(x, y)$ sticks on the west to $f(W(x, y))$. That is to say, $f$ is valid at $(x, y)$ iff the tile at position $(x, y)$ sticks on the appropriate sides to all the tiles that are at positions adjacent to it. The tiling is valid iff it is valid at every position $(x, y) \in \mathbb{Z}^{2}$. The well-known tiling problem posed by Wang asks the following: Given a tile system $T$, does there exist a valid $T$-tiling of the plane? The tiling problem was first proved undecidable by Berger [11], and the result was improved by Robinson [37]. One can generalize the notion of $T$-tiling of the entire plane by defining a partial $T$-tiling as a partial function $g: \mathbb{Z}^{2} \longrightarrow T$. A partial tiling $g$ is valid on a region $R \subseteq \operatorname{dom}(g)$ iff, for all $\left(x_{1}, y_{1}\right),\left(x_{2}, y_{2}\right) \in R$, if $\left(x_{1}, y_{1}\right)$ abuts $\left(x_{2}, y_{2}\right)$ on the north (east, south, west), then $g\left(x_{1}, y_{1}\right)$ sticks to $g\left(x_{2}, y_{2}\right)$ on the north (east, south, west). The partial tiling $g$ is called valid iff it is valid on the entire $\operatorname{dom}(g)$.

A path is a function $P: I \longrightarrow \mathbb{Z}^{2}$, where $I$ is a set of consecutive integers and for all $(i, i+1 \in I), P(i)$ and $P(i+1)$ are adjacent. That is, a path is a sequence of adjacent positions on the plane. For all $i \in I$, we denote $P(i)$ as $\left(x_{i}, y_{i}\right)$. We say that $(x, y)$ is on $P$ iff $(x, y) \in \operatorname{range}(P)$. For all tile systems $T$, a $T$-tiled path is a pair $(P, r)$, where $P$ is a path and $r$ is a function $r$ : range $(P) \longrightarrow T$.

A $T$-tiled path $(P, r)$ is a $T$-ribbon iff

(a) $P$ is one-to-one, and

(b) for all $(i, i+1 \in \operatorname{dom}(P))$, if $\left(x_{i+1}, y_{i+1}\right)$ abuts $\left(x_{i}, y_{i}\right)$ on the north (south, east, west), then $r\left(x_{i+1}, y_{i+1}\right)$ sticks to $r\left(x_{i}, y_{i}\right)$ on the north (south, east, west).

Informally, a tiled path is a ribbon iff it does not cross itself and the glue between tiles at consecutive positions along the path match.

A $T$-ribbon $(P, r)$ is a $T$-zipper iff for all $(x, y),\left(x^{\prime}, y^{\prime}\right)$ on $P$, if $\left(x^{\prime}, y^{\prime}\right)$ abuts $(x, y)$ on the north (south, east, west), then $r\left(x^{\prime}, y^{\prime}\right)$ sticks to $r(x, y)$ on the north (south, east, west). Note that the notion of a zipper is more restrictive than the notion of a ribbon in that a zipper requires all of its tiles (consecutive or not) in adjacent positions to stick.

A directed tile system is a pair $(T, d)$, where $T$ is a tile system and $d: T \longrightarrow$ $\{N, E, S, W\}$ is a function from the tile system to the direction functions. A directed tile system can be thought of as a tile system in which each tile has an arrow painted on it pointing north, east, south, or west. 
A $(T, d)$-directed tiled path is a pair $(P, r)$, where $(P, r)$ is a $T$-tiled path and for all $(i, i+1 \in \operatorname{dom}(P))$ we have that $\left(x_{i+1}, y_{i+1}\right)=d\left(r\left(x_{i}, y_{i}\right)\right)\left(x_{i}, y_{i}\right)$. A directed tiled path is a tiled path in which each tile points to its successor on the path. If $(P, r)$ is a $(T, d)$-directed tiled path and $(P, r)$ is a $T$-ribbon (zipper), then $(P, r)$ is a $(T, d)$-directed ribbon (zipper).

A path, (directed) tiled path, (directed) ribbon, or (directed) zipper is called finite, semi-infinite, or infinite iff the domain of the associated path is finite, has a least or greatest element and is infinite, or is $\mathbb{Z}$, respectively.

To prove the undecidability of existence of infinite ribbons, we shall first prove the undecidability of existence of infinite directed zippers. Afterwards, a "ribbon motif" construction will be employed to prove the result for ribbons: the construction simulates a directed zipper by a ribbon motif of smaller tiles that goes around the contours of the zipper tiles, and it uses geometry to simulate zipper-tile glues.

In order to prove the undecidability of existence of infinite directed zippers, we shall use the undecidability of the tiling problem in conjunction with a sandwich construction that makes use of the existence of a directed tile system with the socalled strong plane-filling property.

Definition 2.1. A directed tile system $(T, d)$ has the strong plane-filling property iff

(i) there exists an infinite $(T, d)$-directed zipper, and

(ii) for all infinite $(T, d)$-directed zippers $(P, r)$, for all natural numbers $n$ there exists $(x, y)$ such that $(x+i, y+j)$ is on $P$ for $i=0,1,2, \ldots, n$ and $j=$ $0,1,2, \ldots, n$.

If a directed zipper (or ribbon or path) $(P, r)$ satisfies the property in Definition 2.1(ii), we also say that it covers arbitrarily large squares.

Definition 2.1 states that in a directed tile system with the strong plane-filling property any infinite directed zipper is forced to be plane-filling (by filling arbitrarily large squares) and, moreover, that such an infinite directed zipper always exists.

3. A directed tile system with the strong plane-filling property. An essential element of our subsequent proofs will be the main result of this section, Theorem 3.1, namely the construction of a directed tile system that satisfies the strong plane-filling property of Definition 2.1. In fact, our directed tiles satisfy an even stronger requirement than (ii): any infinite directed tiled path is either planefilling or it has a glue mismatch between two of its tiles. In particular, using our tiles, an infinite directed tiled path may not even form a loop without encountering a glue mismatch along the way. Consequently, every infinite directed tiled path that does not fill the plane must necessarily contain two neighboring tiles with a glue mismatch. The constructed tiles therefore satisfy a stronger version of Definition 2.1, thus satisfying a stronger property than the original plane-filling property defined in $[26,27]$.

In [27], any infinite directed tiled path was forced to be plane-filling unless there was a mismatch of glues inside the $3 \times 3$ neighborhood of some tile of the path. As described in subsections 3.2 and 3.3, directed tiles with this weaker plane-filling property were explicitly constructed by augmenting Robinson's aperiodic tiles [37] with directions. With these tiles, all directed tiled paths consisting of tiles without errors in their $3 \times 3$ neighborhood formed self-similar plane-filling curves of the kind used by Hilbert [24] to show that the unit square is a continuous image of the unit segment. The constructed tiles filled arbitrarily large squares recursively, by first filling the individual quadrants of a square. The process was guided by the direction associated 
with each tile, which forced the construction to proceed along the self-similar Hilbert curve that traversed each quadrant of a square and linked the quadrants to each other.

In the present application we need the stronger variant of the plane-filling property introduced in Definition 2.1. In our case the infinite directed tiled path must be forced to be plane-filling even under the weak assumption that there is no glue mismatch between any two tiles belonging to it. It turns out that such a directed tile system is obtained by taking $3 \times 3$ blocks of the tiles in [27], as described in subsection 3.4. For this particular set of tiles, our $3 \times 3$ block construction ensures that the weak requirement of absence of glue mismatches between any two tiles belonging to the directed tiled path is sufficient to determine its uniqueness. Namely, we can prove that the constructed directed tile system has the property that, starting with any arbitrary tile, the only way to build an infinite error-free directed zipper is by forming a Hilbert curve that covers arbitrarily large squares.

3.1. Generalized tile systems. We begin by generalizing the notion of neighborhood from section 2, where the "neighbors" of a position were the positions at its north, east, south, and west.

Definition 3.1. A neighborhood vector is a $k$-tuple

$$
V=\left(\overline{x_{1}}, \overline{x_{2}}, \ldots, \overline{x_{k}}\right), \quad \overline{x_{i}} \in \mathbb{Z}^{2}, \quad 1 \leq i \leq k .
$$

The neighbors of a position $\bar{x} \in \mathbb{Z}^{2}$ are the positions $\bar{x}+\bar{x}_{i}$ for $1 \leq i \leq k$.

The classical von Neumann neighborhood is defined by the vector

$$
V_{v N}=[(0,0),(1,0),(0,1),(-1,0),(0,-1)] .
$$

In the von Neumann neighborhood, each position has five neighbors: itself and its four adjacent positions, as defined in section 2. In fact, all notions defined in section 2 are based on the von Neumann neighborhood.

Another particular neighborhood of interest, the Moore neighborhood, is defined as follows:

$$
V_{M}=[(-1,1),(0,1),(1,1),(-1,0),(0,0),(1,0),(-1,-1),(0,-1),(1,-1)] .
$$

The Moore neighborhood of a position is a $3 \times 3$ block centered at that position, where the eight directions of the compass are used when we refer to the eight surrounding positions.

In contrast, in the generalized sense of Definition 3.1, the neighbors of a position need not be adjacent to it. Under this generalized definition, the neighborhood of a position may even have holes in it; i.e., adjacent positions may not belong to its neighborhood, while positions further away may. For example, $V=[(-2,2),(0,2),(2,2)$, $(-1,1),(1,1),(-2,0),(0,0),(2,0),(-1,-1),(1,-1),(-2,-2),(0,-2),(2,-2)]$ defines a checkerboard type of neighborhood, where the neighbors of the center "white" position are all the "white" positions in the $5 \times 5$ square centered at it.

We shall now use the generalized notion of neighborhood to define a generalized tile system and its corresponding notion of valid tiling.

Definition 3.2. A generalized tile system is a triple $(T, V, R)$, where $T$ is a finite set, $V=\left(\bar{x}_{1}, \bar{x}_{2}, \ldots, \bar{x}_{k}\right), \bar{x}_{i} \in \mathbb{Z}^{2}, 1 \leq i \leq k$, is a neighborhood vector, and $R \subseteq T^{k}$ is a $k$-ary relation on $T$.

As usual, a (partial) $(T, V, R)$-tiling (shortly $T$-tiling if the other elements are obvious from the context) is a (partial) function $\psi: \mathbb{Z}^{2} \stackrel{o}{\longrightarrow} T$. 
Definition 3.3. $A(T, V, R)$-tiling $\psi$ is valid at $\bar{x} \in \operatorname{dom}(\psi)$ iff there exists $r \in R$ such that, for all $i, 1 \leq i \leq k$, either $\left(\bar{x}+\bar{x}_{i}\right) \in \operatorname{dom}(\psi)$ and $\psi\left(\bar{x}+\bar{x}_{i}\right)=r(i)$ or otherwise $\psi\left(\bar{x}+\bar{x}_{i}\right)$ is undefined.

A $(T, V, R)$-tiling $\psi$ is valid on a region $S \subseteq \operatorname{dom}(\psi)$ iff $\left.\psi\right|_{S}$ is valid at every point $\bar{x} \in S$. We say that $\psi$ is valid iff it is valid on $\operatorname{dom}(\psi)$. If $\psi$ is valid on $\mathbb{Z}^{2}$, then $\psi$ is called a valid $(T, V, R)$-tiling of the plane.

As before, we can define a generalized directed tile system $(T, V, R, d)$, where $(T, V, R)$ is a generalized tile system and $d: T \longrightarrow\{N, E, S, W\}$ is a function from the generalized directed tile system to the direction functions.

3.2. Microtiles. The starting point of our construction of a directed tile system $\left(T_{0}, d_{0}\right)$ with the strong plane-filling property will be a generalized directed tile system $\left(T_{\mu}, V_{\mu}, R_{\mu}, d_{\mu}\right)$ constructed in [27]. The elements of $T_{\mu}$ are called microtiles. Instead of glues, they use labeled arrows to define their correspondence so that arrow heads match arrow tails on the edges of tiles. A $T_{\mu}$-tiling will sometimes be called microtiling.

There are five different types of microtiles in $T_{\mu}$ (Figure 1): (a) single and (b) double crosses, (c) single, (d) double, and (e) mixed arms. The arms can be horizontal or vertical arms. There are also labeled diagonal arrows assigned to microtiles in [27]. They play a role in proofs of Lemmata 3.1 and 3.2. Since these proofs are not reproduced here, we omit the description of the diagonal arrows.

a)



b)

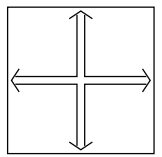

c)

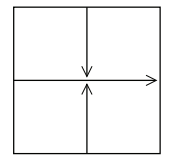

d)



e)

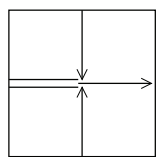

FIG. 1. The five types of microtiles: (a) single cross, (b) double cross, (c) single arm, (d) double arm, and (e) mixed arm. Diagonal arrows are not shown. Reprinted from the Journal of Computer and System Sciences, Volume 48, Jarkko Kari, Reversibility and surjectivity problems of cellular automata, pages 149-182, 1994, with permission from Elsevier.

A cross, either single or double, has its arrow heads labeled from $\{S E, S W, N E$, $N W\}$, but all four arrow heads must have the same label. The principal arrow of an arm (single, double, or mixed) has a label from $\{S E, S W, N E, N W\}$, with the same label on both ends. The side arrows of mixed arms have labels as described in Figure 2. A single or double horizontal arm has its upper (lower) arrow labeled $S X$ (NX, respectively) for $X \in\{E, W\}$. A single or double vertical arm has its left (right) arrow labeled $Y E$ ( $Y W$, respectively) for $Y \in\{N, S\}$. To conclude the description of microtiles, we note that more labels will be described in the next section.
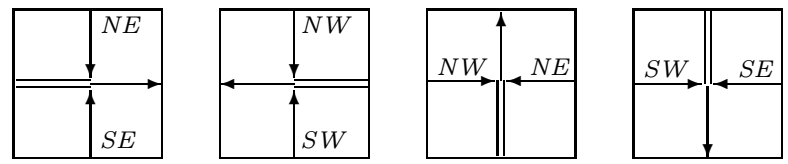

FIG. 2. The possible labels of the two side arrows on a mixed arm. Reprinted from the Journal of Computer and System Sciences, Volume 48, Jarkko Kari, Reversibility and surjectivity problems of cellular automata, pages 149-182, 1994, with permission from Elsevier.

Definition 3.4 (see [27]). A microtile system is a generalized directed tile system $\left(T_{\mu}, V_{\mu}, R_{\mu}, d_{\mu}\right)$, where 


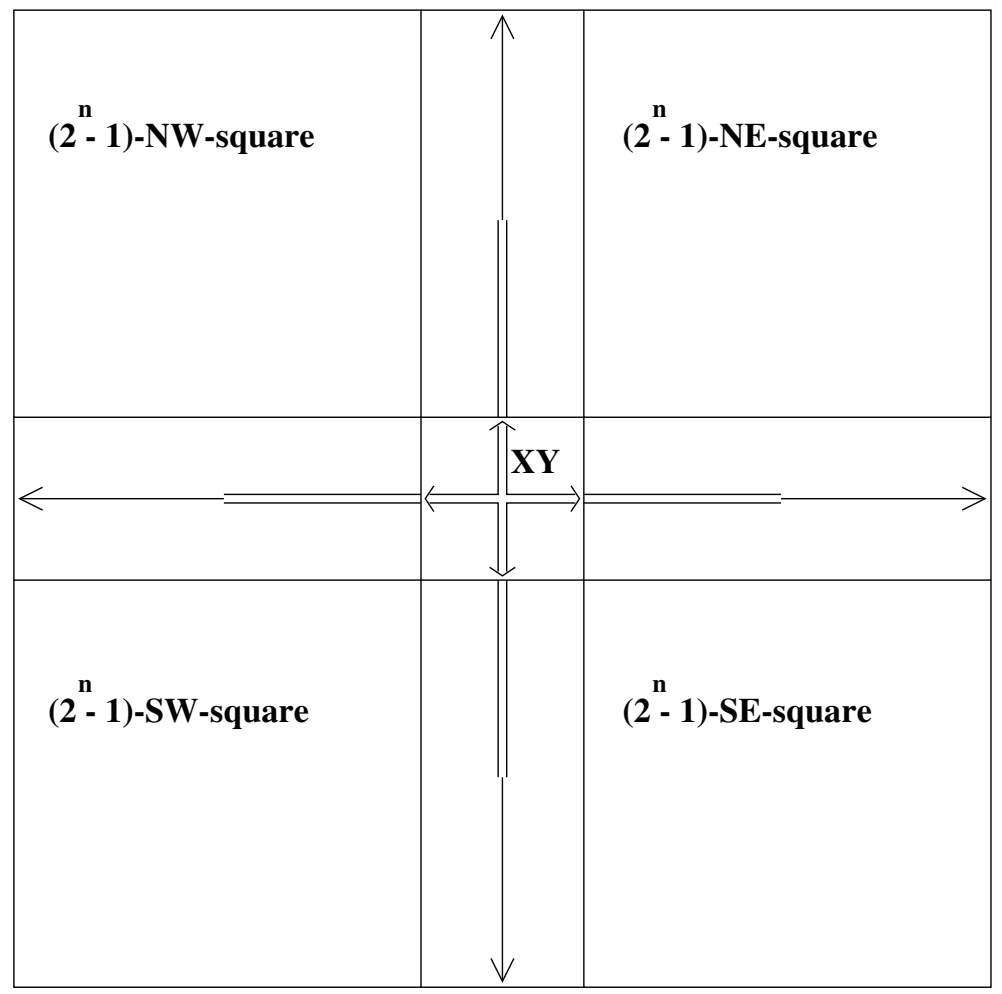

FIG. 3. Constructing the $\left(2^{n+1}-1\right)-X Y$-square. Reprinted from the Journal of Computer and System Sciences, Volume 48, Jarkko Kari, Reversibility and surjectivity problems of cellular automata, pages 149-182, 1994, with permission from Elsevier.

- $T_{\mu}$ is the set of tiles with labeled arrows described above;

- $V_{\mu}$ is the Moore neighborhood;

- $R_{\mu}$ is the set of all $v \in T_{\mu}^{9}$ such that in the $3 \times 3$ tiling $\left\{\left(V_{\mu}(i), v(i)\right) \mid 1 \leq i \leq\right.$ 9\} all arrow heads meet on the edges of tiles matching arrow tails with the same labels and vice versa.

The labeling of microtiles described above allows that, for each $n \in \mathbb{N}$, four special squares called $\left(2^{n}-1\right)-X Y$-squares can be defined recursively.

Definition 3.5. A ( $\left.2^{n}-1\right)-X Y$-square, $n \geq 1, X Y \in\{N E, N W, S E, S W\}$, is a microtiling $f_{X Y}: S_{n} \longrightarrow T_{\mu}$, where $S_{n}=\left\{(x+i, y+j) \mid 1 \leq i, j \leq 2^{n}-1\right\}$ for some $(x, y) \in \mathbb{Z}^{2}$. It is iteratively constructed in the following way.

A single cross labeled $X Y$ is a 1-XY-square. A $\left(2^{n+1}-1\right)-X Y$-square consists of four $\left(2^{n}-1\right)-X^{\prime} Y^{\prime}$-squares, $X^{\prime} Y^{\prime} \in\{N W, N E, S W, S E\}$, ordered as in Figure 3. These squares are separated by a double cross labeled $X Y$ at the position $\left(x+2^{n}, y+2^{n}\right)$, called the central cross of the square, and rows of arms radiating from it. The arms are double near the central cross, but halfway the mixed arms make them single.

The iterative construction is illustrated in Figure 3. By the construction of a microtile system, all $\left(2^{n}-1\right)-X Y$-squares are valid microtilings. Figure 4 provides an example of a $7-X Y$-square. We may sometimes refer to a $\left(2^{n}-1\right)-X Y$-square as $\left(2^{n}-1\right)$-square if $X Y$ is arbitrary. Proofs of the following results can be found in [27].

Lemma 3.1 (see [27]). Let $f: S^{(1)} \longrightarrow T_{\mu}, g: S^{(2)} \longrightarrow T_{\mu}$ be two $\left(2^{n}-1\right)$-squares with the same $n$. If $\left.f\right|_{S^{(1)} \cap S^{(2)}}=\left.g\right|_{S^{(1)} \cap S^{(2)}}$ and there exists $(x, y) \in S^{(1)} \cap S^{(2)}$ such 


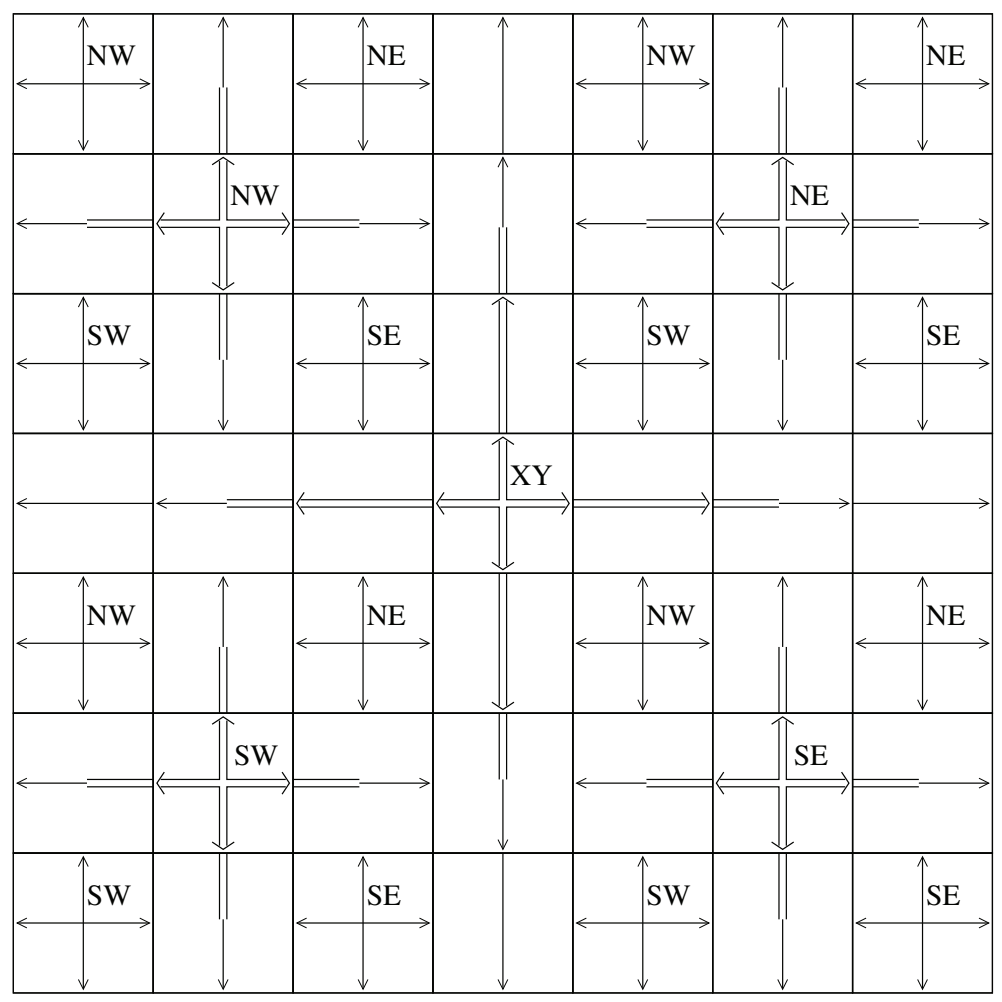

FIG. 4. The 7-XY-square with the labels on the crosses. Only the principal arrows of the arms are drawn. Reprinted from the Journal of Computer and System Sciences, Volume 48, Jarkko Kari, Reversibility and surjectivity problems of cellular automata, pages 149-182, 1994, with permission from Elsevier.

that $f(x, y)$ is a single cross, then $S^{(1)}=S^{(2)}$.

In other words, if $S^{(1)}$ and $S^{(2)}$ have a nonempty common overlap containing a single cross, then they are identical.

Lemma 3.2 (see [27]). Let $f: \mathbb{Z}^{2} \longrightarrow T_{\mu}$ be a microtiling of the plane and assume there exists a $\left(2^{n}-1\right)-X Y$-square $f_{X Y}: S_{n} \longrightarrow T_{\mu}$ such that $\left.f\right|_{S_{n}}=f_{X Y}$ and, moreover, $f$ is valid on $S_{n}$. Let $X Y=S W(N W, N E, S E)$. Then

(i) the microtile just outside the $N E$ ( $S E, S W, N W$, respectively) corner of the square is a double cross;

(ii) the row of arms radiating $Y$-wise ( $X$-wise) from this double cross has the length $2^{n}-1$ and consists of $2^{n-1}-1$ horizontal (vertical) double arms, followed by a horizontal (vertical) mixed arm, and then followed by $2^{n-1}-1$ horizontal (vertical) single arms;

(iii) the microtile just outside the $S E$ ( $N E, N W, S W$, respectively) corner is a horizontal arm and the microtile just outside the $N W$ (SW, SE, NE, respectively) corner is a vertical arm.

In Figure 4, for example, the double cross right outside the NE corner of the $3-S W$-square is the double cross microtile labeled $X Y$, with the row of microtiles radiating westwise and southwise as required.

3.3. Minitiles. Another type of tile needed in our construction is a minitile [27]. Each minitile will be a $2 \times 2$ block of microtiles that has exactly one single cross in 
its upper right corner; see Figure 8(center).

Definition 3.6. Consider the microtile system $\left(T_{\mu}, V_{\mu}, R_{\mu}, d_{\mu}\right)$. A minitile is a quadruple $a=\left(\mu_{1}^{a}, \mu_{2}^{a}, \mu_{3}^{a}, \mu_{4}^{a}\right) \in T_{\mu}^{4}$ such that $\mu_{1}^{a}$ is a single cross, $\mu_{2}^{a}, \mu_{3}^{a}, \mu_{4}^{a}$ are not, and the microtiling $\left\{\left((1,1), \mu_{1}^{a}\right),\left((1,0), \mu_{2}^{a}\right),\left((0,0), \mu_{3}^{a}\right),\left((0,1), \mu_{4}^{a}\right)\right\}$ is valid.

Therefore, by the above definition, all the arrow heads and tails between $\mu_{1}^{a}, \mu_{2}^{a}, \mu_{3}^{a}$, $\mu_{4}^{a}$ match. Let $T_{m}$ be a set of minitiles; then a $T_{m}$-tiling will be called minitiling. If $f: \mathbb{Z}^{2} \stackrel{o}{\longrightarrow} T_{m}$ is a minitiling, then its corresponding microtiling $m(f): \mathbb{Z}^{2} \stackrel{o}{\longrightarrow} T_{\mu}$ is defined as follows. If $f(x, y)=a=\left(\mu_{1}^{a}, \mu_{2}^{a}, \mu_{3}^{a}, \mu_{4}^{a}\right)$, then

$$
\begin{aligned}
m(f)(2 x+1,2 y+1) & =\mu_{1}^{a}, \\
m(f)(2 x+1,2 y) & =\mu_{2}^{a}, \\
m(f)(2 x, 2 y) & =\mu_{3}^{a}, \\
m(f)(2 x, 2 y+1) & =\mu_{4}^{a} .
\end{aligned}
$$

Next, directions are added to minitiles. As we restricted ourselves to $2 \times 2$ blocks having exactly one single cross located in the upper right corner, attaching directions to minitiles will amount to attaching directions to single-cross microtiles. Let us describe first the types of directed minitiled paths (or simply paths, if no danger of confusion exists) these directions will define. The paths are constructed recursively through squares of size $2^{n} \times 2^{n}$ minitiles.

Definition 3.7. For each $n \geq 0$ we define recursively an $A_{2^{n}}\left(B_{2^{n}}, C_{2^{n}}, D_{2^{n}}\right)$ -minitiled path as follows. For $n=0$, a minitile with its single cross labeled $X$ is an $X_{1}$-path for $X \in\{A, B, C, D\}$.

For each $n \geq 0, a A_{2^{n+1}}\left(B_{2^{n+1}}, C_{2^{n+1}}, D_{2^{n+1}}\right)$-path is built recursively from four

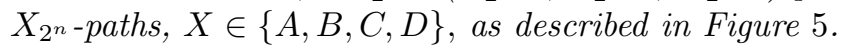

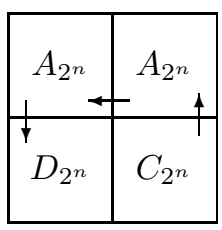

$A_{2^{n+1}}$



$B_{2^{n+1}}$

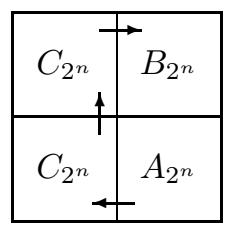

$C_{2^{n+1}}$

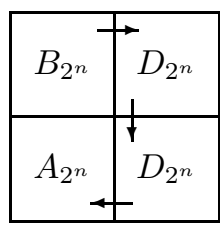

$D_{2^{n+1}}$

FIG. 5. Constructing paths through squares of $2^{n+1} \times 2^{n+1}$ minitiles. Reprinted from the Journal of Computer and System Sciences, Volume 48, Jarkko Kari, Reversibility and surjectivity problems of cellular automata, pages 149-182, 1994, with permission from Elsevier.

For example, the $A_{4}$-path starts in the lower right corner of the square, visits all its minitiles, and ends in the lower left corner (see Figure 6). Paths created using Definition 3.7 form the familiar Hilbert curve. One can easily verify the following result.

Lemma 3.3. For each $n \geq 0$ the $A_{2^{n}}\left(B_{2^{n}}, C_{2^{n}}, D_{2^{n}}\right)$-path starts in the $S E$ (NW, SE, NW, respectively) corner, ends in the $S W$ (NE, NE, SW, respectively) corner, visits all minitiles of the underlying $2^{n} \times 2^{n}$ minitile square, and does not visit any minitiles outside of the underlying square.

Let $f_{X Y}: S_{n+1} \longrightarrow T_{\mu}$ be a $\left(2^{n+1}-1\right)-X Y$-square of microtiles, where $S_{n+1}=$ $\left\{(x+i, y+j) \mid 1 \leq i, j \leq 2^{n+1}-1\right\}$. Then, by the iterative construction in Definition 3.5, those (and only those) microtiles at positions $(x+2 i-1, y+2 j-1), 1 \leq i, j \leq 2^{n}$, are 


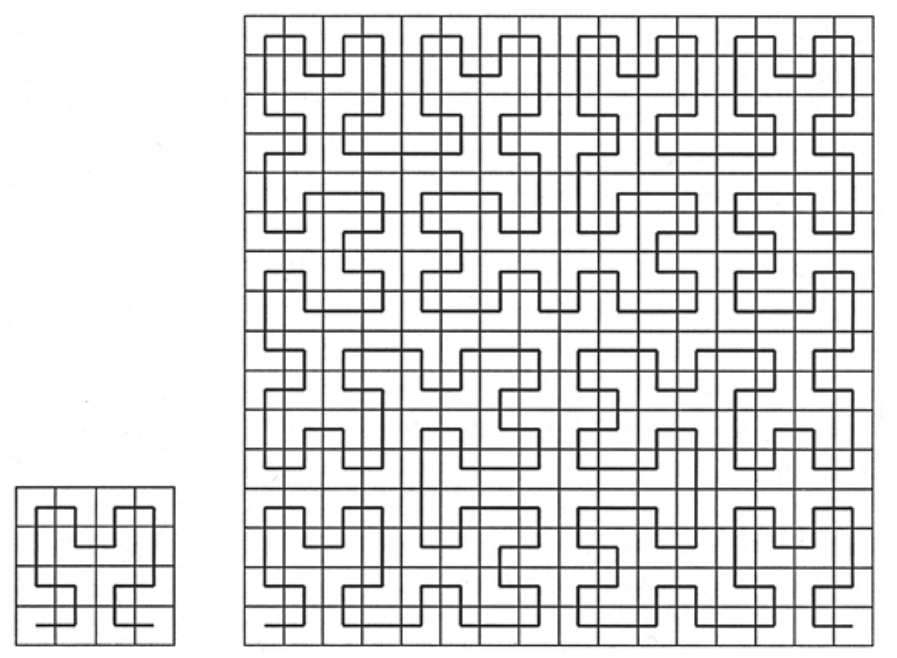

FIG. 6. The paths $A_{4}$ and $A_{16}$. Reprinted from the Journal of Computer and System Sciences, Volume 48, Jarkko Kari, Reversibility and surjectivity problems of cellular automata, pages 149-182, 1994, with permission from Elsevier.

TABLE 1

The labeling of mixed arms. The four rows of the table represent (i) the direction of the principal arrow of the arm, (ii) the label of the principal arrow, (iii) the label of the arrow situated clockwise relative to the principal arrow, and (iv) the label of the arrow situated counterclockwise relative to the principal arrow.

\begin{tabular}{|l|cccc|cccc|cccc|cccc|}
\hline (i) & $E$ & $W$ & $N$ & $S$ & $E$ & $W$ & $N$ & $S$ & $E$ & $W$ & $N$ & $S$ & $E$ & $W$ & $N$ & $S$ \\
(ii) & $A$ & $A$ & $A$ & $A$ & $B$ & $B$ & $B$ & $B$ & $C$ & $C$ & $C$ & $C$ & $D$ & $D$ & $D$ & $D$ \\
(iii) & $C$ & $A$ & $A$ & $D$ & $B$ & $D$ & $C$ & $B$ & $A$ & $C$ & $B$ & $C$ & $D$ & $B$ & $D$ & $A$ \\
(iv) & $A$ & $D$ & $A$ & $C$ & $C$ & $B$ & $D$ & $B$ & $B$ & $C$ & $C$ & $A$ & $D$ & $A$ & $B$ & $D$ \\
\hline
\end{tabular}

single crosses. Since, moreover, $f_{X Y}$ is a valid microtiling, all $2 \times 2$ squares of $S_{n+1}$ with single crosses in their upper right corner form minitiles. The single crosses in the leftmost column and the bottommost row of $S_{n+1}$ can be easily completed by adding another column and a row of microtiles to $f_{X Y}$ so that they again form valid $2 \times 2$ squares of microtiles. Therefore, by Definition 3.6, we obtain a (partial) minitiling $f: \mathbb{Z}^{2} \stackrel{o}{\longrightarrow} T_{m}$ from which the square $f_{X Y}$ arose, i.e., $\left.m(f)\right|_{S_{n+1}}=f_{X Y}$.

If a minitile has its single cross in $S_{n+1}$, we say that it is attached to $S_{n+1}$. Recall that there are exactly $2^{n} \times 2^{n}$ single crosses in $S_{n+1}$; therefore there will be $2^{n} \times 2^{n}=4^{n}$ minitiles attached to it. The directions will be defined in such a way that the path these minitiles form is exactly an $A_{2^{n-}}, B_{2^{n-}}, C_{2^{n-}}$, or $D_{2^{n-p a t h}}$.

In order to control which of the four possible paths the directions define in a specific square, additional labels are assigned to arrows in microtiles [27]. Each arrow (single or double) can be given any label from the set $\{A, B, C, D\}$, the only restrictions we impose on crosses and mixed arms. As usual, in each cross all four arrow heads must have the same label. The central cross of each $\left(2^{n+1}-1\right)$-square will determine the type of path the directions define on the square (see Lemma 3.6). The labeling of mixed arms is crucial for the composition of $A-, B-, C$-, or $D$-squares and is specified in Table 1. As usual, in a valid microtiling the meeting arrow heads and arrow tails must have the same label.

One can verify that the labels in Table 1 correspond exactly to the construction 

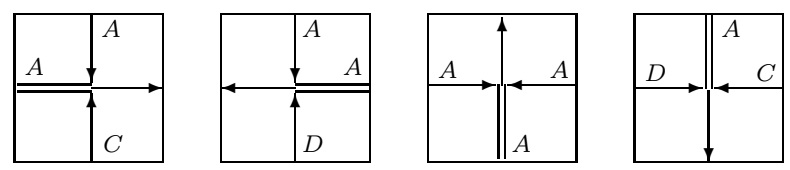

FIG. 7. The labeling of mixed arms whose principal arrow has the label A.

of the paths in Figure 5. For example, the mixed arms in the first four columns in Table 1 are illustrated in Figure 7. The first of these mixed arms has its principal arrow labeled $A$. This indicates that the mixed arm is a connector in a $\left(2^{n+1}-1\right)$ square whose central cross is labeled $A$ and which therefore contains an $A_{2^{n+1}}$-path. The vertical arrow incoming from the north (south) is labeled $A$ (C, respectively). These facts indicate that the $\left(2^{n}-1\right)-N E$-square on top of the mixed arm contains an $A_{2^{n}}$-path, and that the $\left(2^{n}-1\right)-S E$-square below it contains a $C_{2^{n}}$-path, as they

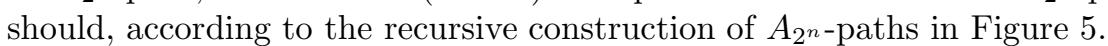

TABLE 2

The definition of directions assigned to minitiles (or to their respective single crosses). Reprinted from the Journal of Computer and System Sciences, Volume 48, Jarkko Kari, Reversibility and surjectivity problems of cellular automata, pages 149-182, 1994, with permission from Elsevier.



The definitions of directions are summarized in Table 2. It has been shown in [27] that if $f_{X Y}: S_{n} \longrightarrow T_{\mu}$ is a $\left(2^{n}-1\right)-X Y$-square, then for each single cross whose Moore neighborhood is contained in $S_{n}$ there exists exactly one rule in Table 2 that can be applied. All the above defined elements forming the directed paths, i.e., the new labels $A, B, C, D$ and the directions of minitiles, are unified together by means of the neighborhood relation $R_{m}$. Now we can complete the formal definition of a minitile system, based on the above described microtile system $\left(T_{\mu}, V_{\mu}, R_{\mu}, d_{\mu}\right)$.

Definition 3.8. A minitile system is a generalized directed tile system $\left(T_{m}, V_{m}\right.$, $\left.R_{m}, d_{m}\right)$ such that

(i) $T_{m}$ is the set of all minitiles as defined in Definition 3.6;

(ii) $V_{m}$ is the Moore neighborhood;

(iii) $R_{m}$ is the set of all 9-tuples $r \in T_{m}^{9}$ such that all the following conditions are met:

(a) the underlying $6 \times 6$ microtiling $m\left(\left\{\left(V_{m}(i), r(i)\right) \mid 1 \leq i \leq 9\right\}\right)$ is valid;

(b) exactly one rule in Table 2 is applicable to the single cross $\mu_{1}^{r(5)}$ of the central minitile $r(5)$;

(c) exactly one of the following four conditions holds: $d_{m}(r(2))=S$, $d_{m}(r(4))=E, d_{m}(r(6))=W, d_{m}(r(8))=N$;

(iv) $d_{m}(a)=d_{\mu}\left(\mu_{1}^{a}\right)$ for each $a=\left(\mu_{1}^{a}, \mu_{2}^{a}, \mu_{3}^{a}, \mu_{4}^{a}\right) \in T_{m}$.

Recall that a 9-tuple $r \in R_{m}$ corresponds to a $3 \times 3$ block of minitiles. The condition (a) states that a necessary condition for the minitiling $f$ to be valid at 

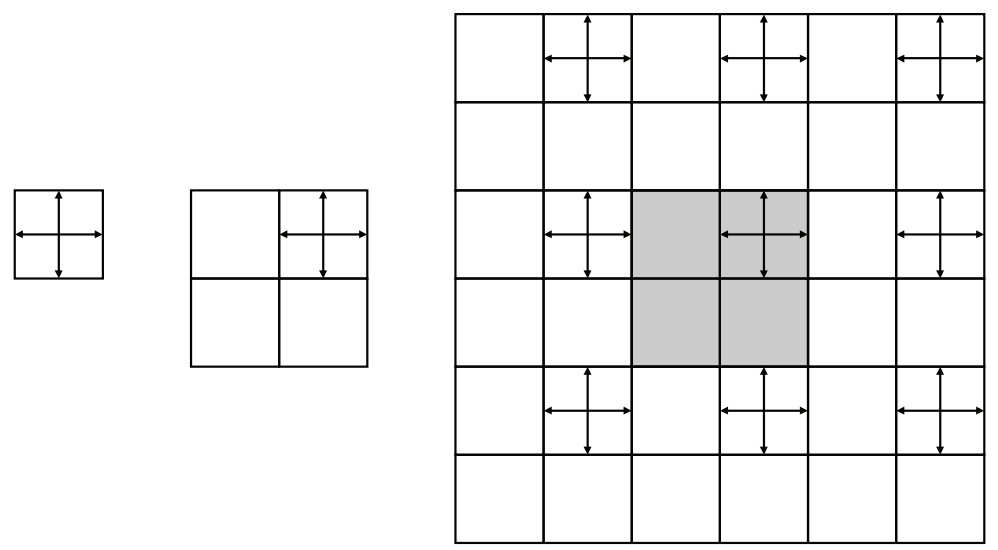

FIG. 8. Left: a microtile; center: a minitile is a $2 \times 2$ block of microtiles having exactly one single cross, in its upper right corner; right: a macrotile (shaded); the nonshaded minitiles define the "glues" of the macrotile.

$(x, y) \in \mathbb{Z}^{2}$ is that its corresponding microtiling $m(f)$ be valid at $(2 x+i, 2 y+j)$ for all $-1 \leq i, j \leq 2$. In other words, inside the $6 \times 6$ block of microtiles corresponding to the $3 \times 3$ Moore neighborhood of $(x, y)$, all arrow heads match meeting arrow tails and their labels match as well, including the new labels $A, B, C, D$.

The condition (b) adds another necessary requirement that the direction of the minitile at $(x, y)$ must be uniquely defined via Table 2 . Finally, by the condition (c) a minitiling is not valid at $(x, y)$ unless exactly one of the four adjacent minitiles at its N, S, E, W has its direction pointing towards it.

Recall that, due to Definition 3.3, if some minitiles in the Moore neighborhood of $(x, y)$ are missing, we consider the minitiling valid at $(x, y)$ if the missing positions can be filled with minitiles such that conditions (iii)(a)-(c) would be met.

3.4. Macrotiles: The $3 \times 3$ block construction. In this section we finalize the construction of a directed tile system $\left(T_{0}, d_{0}\right)$ with the strong plane-filling property. We will use the generalized directed tile system of minitiles $\left(T_{m}, V_{m}, R_{m}, d_{m}\right)$ constructed so far to obtain the directed tile system of macrotiles $\left(T_{0}, d_{0}\right)$. When dealing with $\left(T_{0}, d_{0}\right)$ we revert to the usual notion of tiles sticking by glues as defined in section 2 .

The idea of the construction is as follows. Consider all the validly minitiled Moore neighborhoods as defined by $R_{m}$. With each such 9-tuple, which intuitively is a $3 \times 3$ block of minitiles, we associate a macrotile (see Figure 8 ). The position of the macrotile in a plane is identical with the position of its center minitile. The glues of a macrotile will be the $2 \times 3$ and $3 \times 2$ subblocks of minitiles corresponding to each side. Two adjacent macrotiles will stick if they have the same glues at their abutting edges (see Figure 9).

Definition 3.9. Consider the minitile system $\left(T_{m}, V_{m}, R_{m}, d_{m}\right)$. A macrotile system is a directed tile system $\left(T_{0}, d_{0}\right), T_{0} \subseteq X_{0}^{4}$, where the following hold:

(i) $X_{0}=T_{m}^{6}$.

(ii) $T_{0}$ is constructed as follows. For each validly minitiled Moore neighborhood $t \in R_{m}$, there exists a macrotile $t^{*} \in T_{0}$ with glues

$t_{N}^{*}=(t(1), t(2), t(3), t(4), t(5), t(6))$, $t_{S}^{*}=(t(4), t(5), t(6), t(7), t(8), t(9))$, 


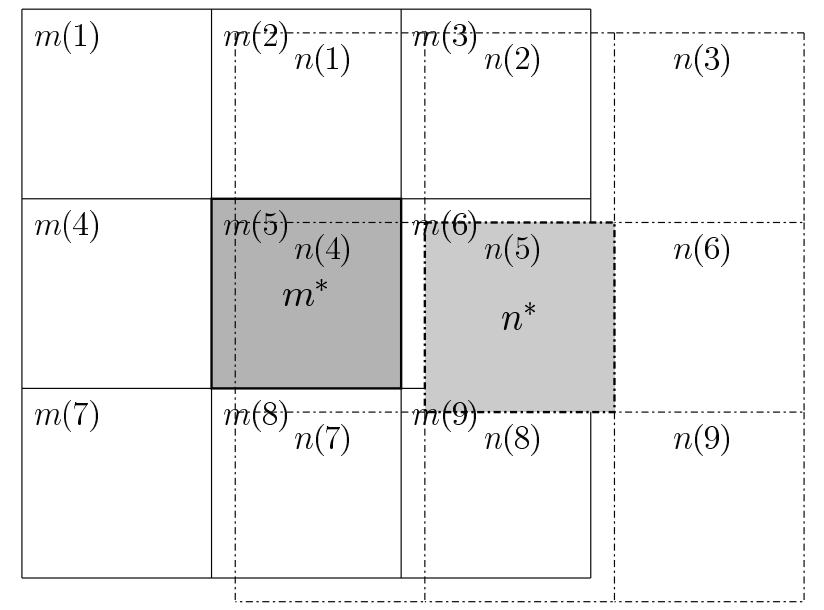

FIG. 9. Two adjacent macrotiles $m^{*}$ and $n^{*}$ (shaded) stick iff their glues at the abutting edges are equal. This means that the overlapping $2 \times 3$ portions of their Moore neighborhoods coincide, i.e., $m(2)=n(1), m(3)=n(2), m(5)=n(4)$, etc.

$$
\begin{aligned}
& t_{E}^{*}=(t(2), t(3), t(5), t(6), t(8), t(9)), \\
& t_{W}^{*}=(t(1), t(2), t(4), t(5), t(7), t(8)) .
\end{aligned}
$$

(iii) $d_{0}\left(t^{*}\right)=d_{m}(t(5))$.

If intuitively a macrotile corresponds to a $3 \times 3$ block of minitiles, its north glue is the $2 \times 3$ top subblock, the south glue is the bottom $2 \times 3$ subblock, the west glue the left $3 \times 2$ subblock, and the east glue the right $3 \times 2$ subblock of minitiles.

The direction of a macrotile $t^{*}$ is the direction of its center minitile $t(5)$ which we will denote also by $c\left(t^{*}\right)$. Similarly as with minitiles, we say that $t^{*}$ is attached to a $\left(2^{n}-1\right)$-square $S_{n} \longrightarrow T_{\mu}$ if the single cross of $c\left(t^{*}\right)$ is located within $S_{n}$.

Two adjacent macrotiles will stick iff the glues on their abutting edges are identical. For example, two macrotiles at positions $(x, y),(x+1, y)$ will stick if, when viewed as $3 \times 3$ blocks centered at $(x, y)$, respectively, $(x+1, y)$, their overlapping minitiles coincide. (See Figure 9.)

Definition 3.10. Consider the macrotile system $\left(T_{0}, d_{0}\right)$ and a $T_{0}$-tiling $f$ : $\mathbb{Z}^{2} \stackrel{\circ}{\longrightarrow} T_{0}$.

(i) The overlap map $o(f): \mathbb{Z}^{2} \stackrel{o}{\longrightarrow} 2^{T_{m}}$ is a function defined as follows. For all $\bar{x} \in \operatorname{dom}(f)$, if $f(\bar{x})=t^{*}$, then $t(i) \in o(f)\left(\bar{x}+V_{m}(i)\right)$.

(ii) The projection map $p(f): \mathbb{Z}^{2} \stackrel{o}{\longrightarrow} T_{m}$ is a function defined as follows: If $(x, y) \in \operatorname{dom}(f)$ and $f(x, y)=t^{*}$, then $p(f)(x, y)=c\left(t^{*}\right)$.

In other words, the overlap map of a set of macrotiles situated on the plane will place at each position all the minitiles that would occupy it if we were to view each macrotile at $(x, y)$ as a $3 \times 3$ block centered at $(x, y)$ and covering a $3 \times 3$ neighborhood around it. The projection map replaces each macrotile at $(x, y)$ with the minitile $c\left(t^{*}\right)$ at its center. Note that, while $\operatorname{dom}(p(f))=\operatorname{dom}(f), \operatorname{dom}(f) \subseteq \operatorname{dom}(o(f))$.

Consider the following special case of the overlap map: for all $(x, y) \in \mathbb{Z}^{2}$, either $o(f)$ is undefined or card $(o(f))=1$. Such a one-to-one overlap map is called a perfect overlap. The following results are straightforward.

LEMMA 3.4. Let $f: \mathbb{Z}^{2} \stackrel{o}{\longrightarrow} T_{0}$ be a $T_{0}$-tiling with $\operatorname{dom}(f)=\left\{(x, y),\left(x^{\prime}, y^{\prime}\right)\right\}$ and the positions $(x, y)$ and $\left(x^{\prime}, y^{\prime}\right)$ be adjacent; then $f(x, y)$ will stick to $f\left(x^{\prime}, y^{\prime}\right)$ iff $o(f)$ is a perfect overlap. 
In other words, two macrotiles situated at adjacent positions will stick (in the gluematch-at-abutting-edges sense defined in section 2) iff the overlap map they define is one-to-one.

Let us introduce the following notation for the set of positions on the plane belonging to the Moore neighborhood of $\bar{x} \in \mathbb{Z}^{2}: N_{M}(\bar{x})=\left\{\bar{x}+V_{M}(i) \mid 1 \leq i \leq 9\right\}$.

Lemma 3.5. Let $f: \mathbb{Z}^{2} \stackrel{o}{\longrightarrow} T_{0}$ be a $T_{0}$-tiling and let $\bar{x}, \bar{y} \in \operatorname{dom}(f)$. Further let $f$ contain a $T_{0}$-ribbon $(P, r)$ such that $\{\bar{x}, \bar{y}\} \subseteq \operatorname{range}(P)$ and

$$
N_{M}(\bar{x}) \cap N_{M}(\bar{y}) \subseteq \bigcap_{\bar{z} \in \operatorname{range}(P)} N_{M}(\bar{z}) .
$$

Then the overlap map o(f $\left.\left.\right|_{\{\bar{x}, \bar{y}\}}\right)$ is perfect.

Informally, let two macrotiles $x^{*}$ and $y^{*}$ be connected with a (short) ribbon such that the intersection of the Moore neighborhoods of $x^{*}$ and $y^{*}$ is included in (and hence is equal to) the intersection of the Moore neighborhoods of all tiles in the ribbon. Then, by Lemma 3.4, each two adjacent tiles in the ribbon have perfect overlap. By transitivity, we deduce that the intersection of overlap maps of all the tiles in the ribbon is one-to-one and hence so is the common overlap map of $x^{*}$ and $y^{*}$.

The following lemma states that each infinite directed zipper of macrotiles covers arbitrarily large squares.

Lemma 3.6. Let $n \in \mathbb{N}$ and let $(P, r)$ be a $T_{0}$-directed zipper, $P: I \longrightarrow \mathbb{Z}^{2}$, $r$ : range $(P) \longrightarrow T_{0}$, such that there exists $i \in I$ with $\min (I)+4^{n} \leq i \leq \max (I)-4^{n}$. Denote $P(i)=\left(x_{i}, y_{i}\right)$ and $r\left(x_{i}, y_{i}\right)=t^{*}$. Then the following hold:

(i) There exists a $\left(2^{n+1}-1\right)$-square $f_{X Y}: S_{n+1} \longrightarrow T_{\mu}$ such that $\left(2 x_{i}+1,2 y_{i}+\right.$ 1) $\in S_{n+1}, S_{n+1} \subseteq \operatorname{dom}(m(p(r)))$ and $f_{X Y}=\left.m(p(r))\right|_{S_{n+1}}$.

(ii) Let $Q=\left\{(k, l) \in \mathbb{Z}^{2} \mid(2 k+1,2 l+1) \in S_{n+1}\right\}$. If the central cross of $S_{n+1}$ has label $A(B, C, D)$, then $p\left(\left.r\right|_{Q}\right)$ is an $A_{2^{n}}\left(B_{2^{n}}, C_{2^{n}}, D_{2^{n}}\right.$, respectively)-path.

(iii) The overlap map o $\left(\left.r\right|_{Q}\right)$ is a perfect overlap.

Informally, consider a directed zipper of macrotiles that passes through a macrotile $t^{*}$ and extends for at least $4^{n}$ positions preceding and the $4^{n}$ positions succeeding the position of $t^{*}$. Then (i) the single cross of the minitile $c\left(t^{*}\right)$ belongs to a $\left(2^{n+1}-1\right)$ square $f_{X Y}: S_{n+1} \longrightarrow T_{\mu}$. Moreover, (ii) the projection of the portion of the directed zipper consisting of the macrotiles attached to $S_{n+1}$ is an $A_{2^{n-}}, B_{2^{n-}}, C_{2^{n-}}$, or $D_{2^{n-}}$ directed minitiled path if the central cross microtile of the square has label $A, B, C$, or $D$, respectively. Last, (iii) the overlap map of that portion of the directed zipper is a perfect overlap.

Proof. The proof is by induction on $n$.

$n=0$. Assume there is no zipper error involving $4^{0}=1$ macrotile before and one macrotile after $t^{*}$. Then the required $2^{n+1}-1=2^{1}-1=1$-square consists of only the single cross at the upper right corner of the minitile $c\left(t^{*}\right)$ and the statement is vacuously true.

Assuming the statement holds for $n-1$, we prove it for $n$. Assume there are no zipper errors involving any of the $4^{n}$ macrotiles that precede or succeed $t^{*}$. By induction hypothesis (I.H.) (i), the minitile $c\left(t^{*}\right)$ is attached to a $\left(2^{n}-1\right)$-square $S^{(1)} \longrightarrow T_{\mu}$ with the required properties. Assume that it is a $\left(2^{n}-1\right)-S W$-square (the other cases are analogous.)

Properties of the square $S^{(1)}$.

Let $Q_{1}=\left\{(k, l) \in \mathbb{Z}^{2} \mid(2 k+1,2 l+1) \in S^{(1)}\right\}$ be the square of macrotiles attached to $S^{(1)}$. By I.H. (iii), the overlap map of $\left.r\right|_{Q_{1}}$ is perfect (one-to-one). The underlying microtiling $m\left(o\left(\left.r\right|_{Q_{1}}\right)\right)$ covers $S^{(1)}$ and the Moore neighborhood of all its 


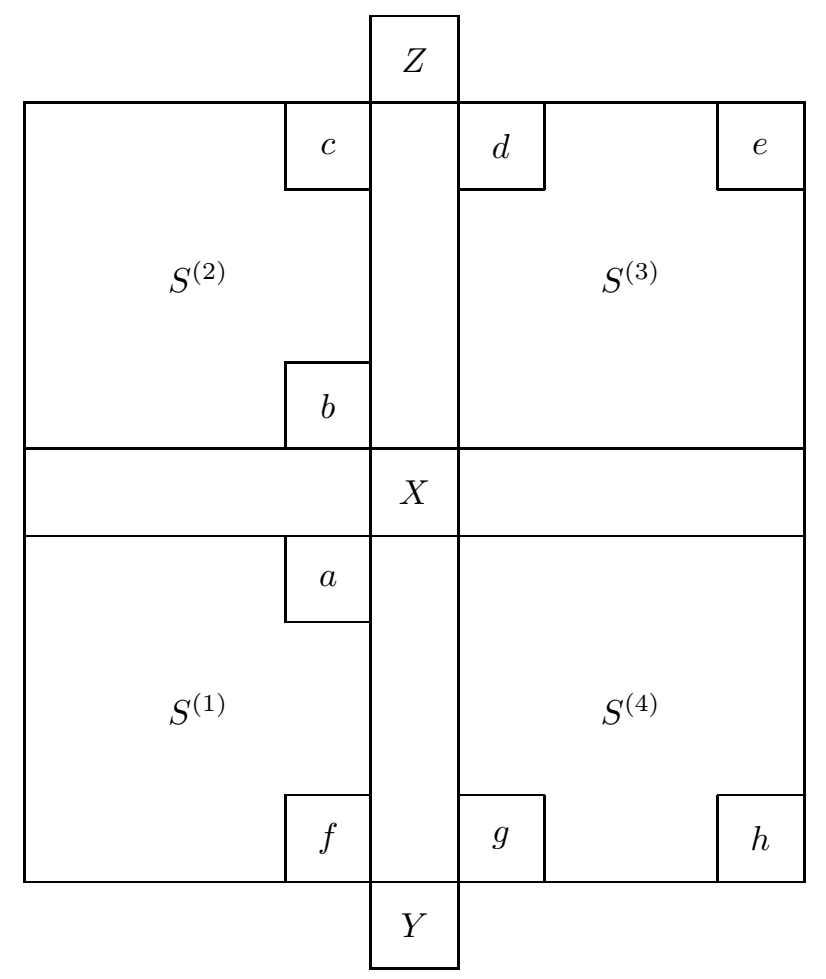

FIG. 10. The $\left(2^{n+1}-1\right)$-square constructed during the proof of Lemma 3.6. Reprinted from the Journal of Computer and System Sciences, Volume 48, Jarkko Kari, Reversibility and surjectivity problems of cellular automata, pages 149-182, 1994, with permission from Elsevier.

tiles (actually, it is even larger) and, by Definitions 3.8 and 3.9 , is valid. Then, by Lemma 3.2, the microtile just outside the upper right corner of $S^{(1)}$ is a double cross, denoted by $X$ in Figure 10. The double cross can have any of the four labels $A, B$, $C, D$. Assume it has label $C$.

By Lemma 3.2 again, the microtiles exactly on top of $S^{(1)}$ are horizontal arms (first double arms, then one mixed arm, then single arms) radiating westwise, with the same label $C$. Then, by Table 1 , the lower edge of the mixed arm has a side arrow labeled $C$ (column 10, row 4 of the table). Then the central cross of $S^{(1)}$ has the same label $C$, due to the column of arms radiating from it northwise to the mentioned mixed arm (see Definition 3.5). This means the path through $S^{(1)}$ is, by I.H. (ii), a $C$-path which, by Lemma 3.3, starts at a single cross $f$ and ends at a single cross $a$ (Figure 10).

Adding the square $S^{(2)}$.

As $a$ has at its NE a double cross labeled $C$, according to Table 2, the direction of $a$ is $\mathrm{N}$. This means that the path of macrotiles, passing through the macrotile $a^{*}$ with the center single cross $a$, will proceed to $b^{*}$. Denote by $b$ the single cross of $c\left(b^{*}\right)$ (Figure 10).

Recall that for the macrotile $t^{*}$ attached to $S^{(1)}$ we assumed that it is preceded and succeeded by at least $4^{n}$ macrotiles on the path $P$. Since there are exactly $4^{n-1}$ macrotiles attached to $S^{(1)}$, the distance of $t^{*}$ and $b^{*}$ on the path $P$ is at most $4^{n-1}$, by Lemma 3.3. Hence the I.H. can be applied to $b^{*}$ because the number of macrotiles preceding and succeeding $b^{*}$ on $P$ is at least $4^{n}-4^{n-1}=3 \cdot 4^{n-1}>4^{n-1}$. By I.H. (i), 
$b$ belongs to a $\left(2^{n}-1\right)$-square $S^{(2)} \longrightarrow T_{\mu}$.

Observe that $a$ is not in $S^{(2)}$ (otherwise, by Lemma 3.1, $S^{(1)}$ and $S^{(2)}$ would coincide, which is impossible as $b$ is in $S^{(2)}-S^{(1)}$ ). Hence $S^{(1)}$ and $S^{(2)}$ are disjoint. By I.H. (ii), $S^{(2)}$ contains an $A-, B-, C$-, or $D$-path. As $c\left(b^{*}\right)$ is the first minitile of the path, and $b$ is located just above $a$, by Lemma 3.3 the path must be either an $A$-path or $C$-path. In both cases $b$ is located in the $\mathrm{SE}$ corner of $S^{(2)}$, and we conclude that $S^{(2)}$ is above $S^{(1)}$.

As $S^{(2)}$ has all the properties conferred by I.H., we repeat for $S^{(2)}$ the steps previously made for $S^{(1)}$. In particular, let $Q_{2}=\left\{(k, l) \in \mathbb{Z}^{2} \mid(2 k+1,2 l+1) \in S^{(2)}\right\}$. By I.H. (iii), the overlap map $o\left(\left.r\right|_{Q_{2}}\right)$ of the macrotiles attached to $S^{(2)}$ is one-to-one. Hence, the microtiling $m\left(o\left(\left.r\right|_{Q_{2}}\right)\right)$ covering $S^{(2)}$ and its Moore neighborhood is valid.

By definition of mixed arms in Figure 2, the mixed arm under $S^{(2)}$ has an upper edge with a side arrow labeled $N W$. As there is a column of arms connecting this arrow with the central microtile of $S^{(2)}$, we have that $S^{(2)}$ is a $\left(2^{n}-1\right)-N W$-square. By Lemma 3.2 we have to the right of $S^{(2)}$ a column of vertical arms with a mixed arm in the middle. By Table 1, the left edge of this mixed arm has a side arrow labeled $C$, and hence the central cross of $S^{(2)}$ is also labeled $C$. By I.H. (ii), the path through $S^{(2)}$ is a $C$-path. By Lemma 3.3, the path enters through $b^{*}$ and exits through $c^{*}$ (Figure 10).

We know already that, when taken separately, the overlap maps of the partial tilings with macrotiles attached to $S^{(1)}$, respectively, $S^{(2)}$, are one-to-one. When considering the overlap map associated with $S^{(1)}$ and $S^{(2)}$, we have to inspect the cases when there are two macrotiles, $x^{*}$ attached to $S^{(1)}$ and $y^{*}$ attached to $S^{(2)}$, such that their Moore neighborhoods overlap. The possible cases are those described in Figure 11 and the symmetrical ones. Recall that, by Lemma 3.3, the zipper $(P, r)$ visits all the macrotiles attached to $S^{(1)} \cup S^{(2)}$. Hence any adjacent pair of them sticks and any tiled path forms a ribbon. Then, in each of the cases in Figure 11 the figure also shows the short ribbon required by Lemma 3.5. (These ribbons have nothing to do with the zipper $(P, r)$.) Hence, by Lemma 3.5, the common overlap map of $x^{*}$ and $y^{*}$ is perfect. This further implies that the overlap map $o\left(\left.r\right|_{Q_{1} \cup Q_{2}}\right)$ of all the macrotiles attached to $S^{(1)}$ and $S^{(2)}$ is one-to-one.
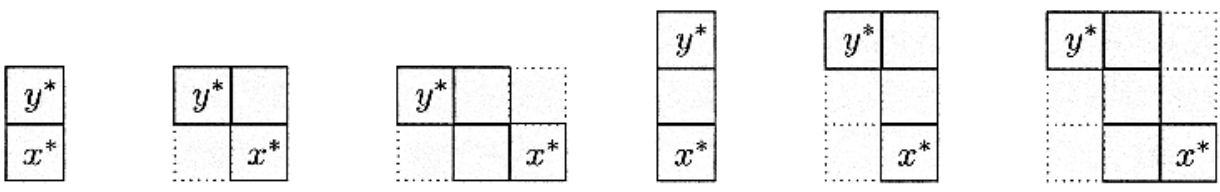

FIG. 11. Pairs of macrotiles attached to $S^{(1)}$ and $S^{(2)}$ with nonempty common overlap.

Adding the square $S^{(3)}$.

By Lemma 3.2(ii) applied to $S^{(2)}$, there is a row of vertical arms labeled $C$ to the right of $S^{(2)}$ which ends just to the right of the single cross $c$. By Lemma 3.2(iii) $Z$ must be a horizontal arm whose lower edge has its side arrow labeled $C$. As $Z$ is the NE neighbor of $c$, by Table 2, the direction of $c$ is $E$. We can reason similarly as in the case of tile $b$ in $S^{(2)}$ to conclude that $d$ is the first single cross in a $B$-path through a $\left(2^{n}-1\right)-N E$-square $S^{(3)} \longrightarrow T_{\mu}$ situated at the right side of $S^{(2)}$. The path ends at tile $e$ (Figure 10).

Reasoning as in the case of $S^{(1)}$ and $S^{(2)}$ we can conclude that the overlap map of macrotiles associated with $S^{(2)}$ and $S^{(3)}$ is one-to-one. To conclude that the overlap 
map associated with $S^{(1)}$ and $S^{(2)}$ and $S^{(3)}$ is one-to-one, we have to consider pairs of macrotiles, $x^{*}$ attached to $S^{(1)}$ and $y^{*}$ attached to $S^{(3)}$, such that their Moore neighborhoods overlap. One can easily verify that there are at most nine such pairs. Similarly as in the case of common overlap of $S^{(1)}$ and $S^{(2)}$, for each of these pairs there exists a short ribbon satisfying the requirements of Lemma 3.5. By reasoning similar to the previous case, the overlap map of $x^{*}$ and $y^{*}$ is one-to-one and so is the common overlap map of macrotiles attached to $S^{(1)} \cup S^{(2)} \cup S^{(3)}$.

Adding the square $S^{(4)}$.

Consider the overlap map of macrotiles attached to $S^{(1)}, S^{(2)}$, and $S^{(3)}$ which forms a valid minitiling. Let $f^{*}$ be the macrotile with the center single cross $f$, where the $C$-path through $S^{(1)}$ starts. The overlap map extends one minitile to the east and south of $f^{*}$, hence covering both microtiles $g$ and $Y$. By Lemma 3.2, $Y$ is a horizontal arm whose upper edge has a side arrow labeled $C$. As $Y$ is the SW neighbor of the single cross $g$, it implies, by Table 2 , that $g$ has to have direction $W$.

Moreover, by the nonexistence of a zipper error on the path segment considered, and the definition of the overlap map, there is no tiling error in the overlap map of $S^{(1)}, S^{(2)}$, and $S^{(3)}$. Recall that, by Definition 3.8 , in a valid minitiling each single cross has only one other single cross pointing towards it. Consequently, $g$ is the unique predecessor of $f$ on the path; therefore $g^{*}$ uniquely precedes $f^{*}$.

According to I.H. (i), $g$ is known to belong to a $\left(2^{n}-1\right)$-square $S^{(4)} \longrightarrow T_{\mu}$. In the same way as before, we conclude that $S^{(4)}$ is situated at the right side of $S^{(1)}$, and the path through $S^{(4)}$ is an $A$-path starting at $h$ and ending at $g$ that visits all its single crosses. We can also reason as before to prove that the overlap map of macrotiles involved in $S^{(1)}, S^{(2)}, S^{(3)}$, and $S^{(4)}$ is one-to-one.

Conclusion.

Altogether, by Definition 3.5 and Lemma 3.2 , the squares $S^{(1)}, S^{(2)}, S^{(3)}$, and $S^{(4)}$ form a $\left(2^{n+1}-1\right)$-square $f_{X Y}: S_{n+1} \longrightarrow T_{\mu}$. All its single crosses are visited by the projection of the path and the macrotile $t^{*}$ is on this path, as required in the statement (i) of the lemma.

Furthermore, by Definition 3.7, the $A$-, $C$-, $C$-, and $B$-paths through $f_{X Y}$ form a $C_{2^{n+1}}$-path. As we have started with the assumption that the central cross $X$ of $S_{n+1}$ is labeled $C$, the statement (ii) of the lemma holds, too.

Finally, we have also shown that the common overlap map of the partial macrotiling with macrotiles attached to $S^{(1)}, S^{(2)}, S^{(3)}$, and $S^{(4)}$ is a perfect overlap. This verifies the statement (iii) of the lemma and concludes the induction step. The other cases ( $X$ labeled by $A, B, D$, and $S^{(1)}$ having other label than $S W$ ) are analogous.

THEOREM 3.1. There exists a directed macrotile system $\left(T_{0}, d_{0}\right)$ with the strong plane-filling property.

Proof. Consider the macrotile system $\left(T_{0}, d_{0}\right)$ from Definition 3.9. Observe that there exists an infinite directed zipper of macrotiles from $T_{0}$, namely the zipper $(P, r)$ constructed inductively in the proof of Lemma 3.6. Moreover, by the statement of Lemma 3.6(i), any infinite path following the directions, which in addition has no zipper errors, covers arbitrarily large squares.

4. Undecidability of existence of infinite ribbons. We shall now use Theorem 3.1 to prove the undecidability of existence of an infinite directed zipper.

THEOREM 4.1. The set $\{(T, d) \mid(T, d)$ is a directed tile system and there exists an infinite $(T, d)$-directed zipper $\}$ is undecidable.

Proof. Reduce the undecidable tiling problem to our problem. 


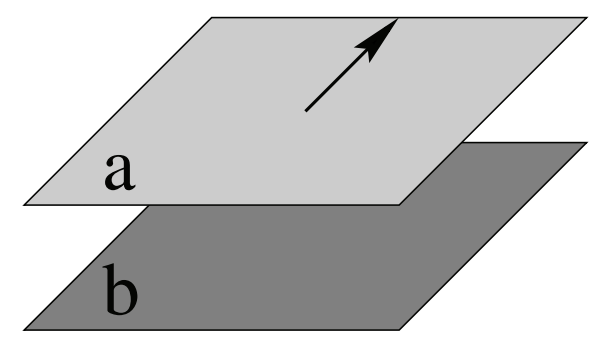

FIG. 12. A sandwich tile $\sigma(a, b)$ consists of a directed tile $a \in T_{0}$ placed on top of a tile $b \in T_{1}$. The direction of $\sigma(a, b)$ is $d(\sigma(a, b))=d_{0}(a)$, the direction of its top tile.

By Theorem 3.1 there exists a directed tile system $\left(T_{0}, d_{0}\right)$ with the strong planefilling property.

Let $T_{1}$ be a tile system. Consider the following "sandwich tiles," each consisting of a tile from $T_{0}$ placed on top of a tile from $T_{1}$. That is, create a new directed tile system $(T, d)$ of sandwich tiles such that $T=\left\{\sigma(a, b) \mid a \in T_{0}\right.$ and $\left.b \in T_{1}\right\}$, where, for all $a=\left(a_{N}, a_{E}, a_{S}, a_{W}\right) \in T_{0}$ and $b=\left(b_{N}, b_{E}, b_{S}, b_{W}\right) \in T_{1}$, the sandwich tile $\sigma(a, b)=\left(\left(a_{N}, b_{N}\right),\left(a_{E}, b_{E}\right),\left(a_{S}, b_{S}\right),\left(a_{W}, b_{W}\right)\right)$ and the direction of the sandwich tile is $d(\sigma(a, b))=d_{0}(a)$ (see Figure 12).

Hence, a sandwich tile $\sigma(a, b)$ sticks on the north (east, south, west) to $\sigma\left(a^{\prime}, b^{\prime}\right)$ iff $a$ sticks on the north (east, south, west) to $a^{\prime}$ and $b$ sticks on the north (east, south, west) to $b^{\prime}$.

We will show now that there exists a valid $T_{1}$-tiling of the plane iff there exists an infinite $(T, d)$-directed zipper.

" $\Rightarrow$ " Assume that there exists a valid $T_{1}$-tiling of the plane $f: \mathbb{Z}^{2} \longrightarrow T_{1}$. Consider an infinite $\left(T_{0}, d_{0}\right)$-directed zipper $(P, r)$ (which exists, since $\left(T_{0}, d_{0}\right)$ has the strong plane-filling property). Consider the $T$-tiled path $(P, q)$ of sandwich tiles such that, for all $(x, y)$ on $P, q(x, y)=\sigma(r(x, y), f(x, y))$. Informally, the directed tiled path of sandwich tiles consists of the infinite $\left(T_{0}, d_{0}\right)$-directed zipper on its top and the corresponding partial valid $T_{1}$-tiling $\left.f\right|_{\text {range }(P)}$ on the bottom. It is clear that in fact $(P, q)$ is an infinite $(T, d)$-directed zipper of sandwich tiles.

" $\Leftarrow$ " Assume that there exists an infinite $(T, d)$-directed zipper $(P, q)$ of sandwich tiles. Then, let $(P, r)$ be its top layer; i.e., for all $(x, y)$ on $P, r(x, y)=a$ iff $q(x, y)=$ $\sigma(a, b)$ for some $b \in T_{1}$. Then clearly, $(P, r)$ is an infinite $\left(T_{0}, d_{0}\right)$-directed zipper. Hence, as $\left(T_{0}, d_{0}\right)$ has the strong plane-filling property, $P$ contains "arbitrarily large squares": for all $n$ there exists $(x, y)$ such that, $(x+i, y+j)$ is on $P$ for $i=0,1,2, \ldots, n$ and $j=0,1,2, \ldots, n$. Let $(P, z)$ be the bottom layer of $(P, q)$, i.e., the $T_{1}$-tiled path such that, for all $(x, y)$ on $P, z(x, y)=b$ iff $q(x, y)=\sigma(a, b)$. Then clearly, $(P, z)$ is in fact an infinite $T_{1}$-zipper. It follows that $\operatorname{range}(P)=\operatorname{dom}(z)$ contains arbitrarily large squares and, moreover, for all $n, z: \operatorname{range}(P) \longrightarrow T_{1}$ is a partial tiling valid on a square of size $n$. It now follows from the König infinity lemma [28] that there exists a valid $T_{1}$-tiling of the plane.

Having proved the undecidability of existence of an infinite directed zipper, we shall use this result to show that the existence of an infinite ribbon is also undecidable.

TheOrem 4.2. The set $\{T \mid T$ is a tile system and there exists an infinite $T$-ribbon $\}$ is undecidable.

Proof. Reduce the set of Theorem 4.1 to this set.

Given a directed tile system $(T, d)$, we will construct a tile system $T^{\prime}$ such that there exists an infinite $(T, d)$-directed zipper iff there exists an infinite $T^{\prime}$-ribbon. 




FIG. 13. A ribbon motif with input tile on the west and output tile on the north.

Recall that a zipper differs from a ribbon in that in a ribbon only consecutive tiles are required to have matching glues on abutting edges, while on a zipper even nonconsecutive neighboring tiles have to have matching glues on their abutting edges.

The construction is as follows. For each directed tile $t \in T$, construct three $T^{\prime}$ motifs. A motif is a finite $T^{\prime}$-ribbon $(P, r)$ of special form. We construct these ribbon motifs as follows (see Figure 13).

Each motif is essentially a tiled path that outlines the contours of a square. There are dents and bumps on the north, east, south, and west sides of the motif. In addition, if $(P, r)$ is a motif, then the first position on $P$ is midway along one side of the motif. This side is called the input side of the motif and the tile at the first position is called the input tile of the motif. The last position on $P$ is midway along a side of the motif different from the input side. This side is called the output side of the motif and the tile at the last position is called the output tile of the motif.

Given a directed tile $t \in T$, we construct the three possible motifs with output side $d(t)$. We call these three motifs the "variants" of $t$ (see Figure 14). On each variant, we put a bump on the east (south) side, to encode the glue on the east (south) side of $t$. We also put a dent on the west (north) side, to encode the glue on the west (north) side of $t$. The dents and bumps are designed so that if, for example, $t_{1}$ sticks on the north to $t_{2}$, then the dents on the north of $t_{1}$ variant motifs fit the bumps on the south of $t_{2}$ variants (see Figure 15). If, however, $t_{1}$ does not stick on the north to $t_{2}$, then the north sides of $t_{1}$ variant motifs will overlap the bumps on the south of $t_{2}$ variants. Since overlaps are not allowed in ribbons, if $t_{1}$ does not stick on the north to $t_{2}$, no $T^{\prime}$-ribbon can have a $t_{2}$ variant motif directly north of a $t_{1}$ variant motif.

The glues on tiles in $T^{\prime}$ are chosen so that each tile can occur in exactly one variant motif, and in each variant motif it occurs exactly once. To this aim, the two edges of each ribbon tile connecting it with the preceding (succeeding) tile on a variant motif are labeled so that this is the only sticking that can form along those edges. The only exception to this rule are the input (output) tiles of motifs, which have the edges connecting them to the preceding (succeeding) tiles labeled differently, as detailed below. 

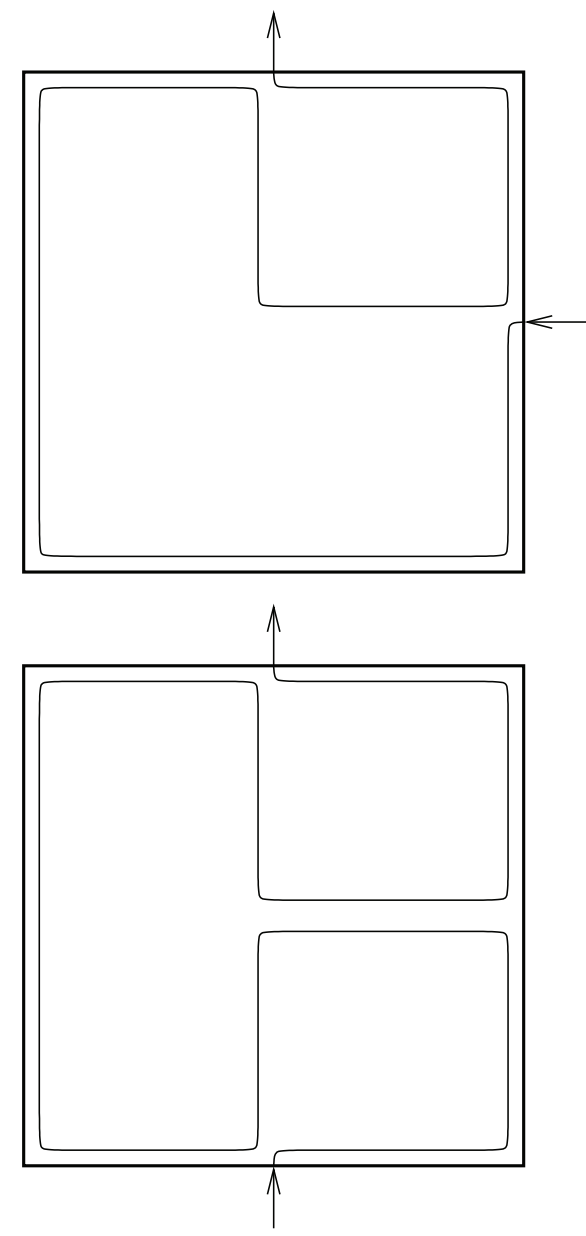

FIG. 14. The two remaining variant motifs corresponding to a directed zipper tile with direction north (the first one is depicted in Figure 13). The bumps and dents are omitted from the variant motifs for clarity.



FIG. 15. The bump-and-dent pair that simulates a glue. Each glue is assigned a unique position on the side of a motif. If the glues on two directed zipper tiles situated at adjacent positions match, then the bump on the first motif will be placed exactly at the necessary position to fit in the dent of the second motif.

Copyright (c) by SIAM. Unauthorized reproduction of this article is prohibited. 
So far, the bumps and dents were used to simulate the glues of zipper tiles. We now simulate the direction of a zipper tile by incorporating it in the glues of the input and output tiles of its variant motifs. We namely use four new glues, West-to-East, East-to-West, North-to-South, and South-to-North as follows. If the direction of a directed zipper tile $t_{1}$ is north, i.e., $d\left(t_{1}\right)=N$, then

(a) the output ribbon-tiles of all three variant motifs of $t_{1}$ will have their north edge labeled South-to-North, and

(b) the input tile of the variant motif of $t_{1}$ with a west (east, south) input side will have its west (east, south) edge labeled West-to-East (East-to-West, South-to-North).

We label in a similar way the appropriate edges of the input and output tiles of other motifs, namely those edges that are meant to connect the motifs to each other. This labeling ensures that the variant motifs will be connected to each other in the proper order dictated by the direction of the originating directed zipper tiles.

Last, we want to ensure that only motifs of the kind described above can form, and that motifs can connect to each other only through their input and output tiles. To this aim, we have two new different "null" glues: null(1) and null(2). We label, for all the above constructed ribbon tiles, the so-far-unlabeled north and west edges with null(1) and the unlabeled east and south edges with null(2). Because null(1) matches only null(1), and null(1) is used only on the west and north edges, the edges of tiles labeled with these glues will not stick to any other tiles along those edges. The same is true for $\operatorname{null}(2)$. These "nonstick" glues ensure that the ribbon will only follow the intended motif and will not fill it in, or stick to anything outside it, except at the input and output tiles.

To summarize, given the directed tile system $(T, d)$ we can construct the tile system $T^{\prime}$ consisting of the ribbon tiles used in all variant motifs of tiles in $T$, as described above.

Assume that there exists an infinite directed $(T, d)$-zipper $(P, r)$. One can easily construct an infinite $T^{\prime}$-ribbon $\left(P^{\prime}, r^{\prime}\right)$. The idea is that, for each position $\left(x_{i}, y_{i}\right)$ on $P$, the tile $r\left(x_{i}, y_{i}\right)$ is replaced by one of its variant motifs. The variant chosen is one with input side opposite $d\left(r\left(x_{i-1}, y_{i-1}\right)\right)$.

Conversely, assume that there exists an infinite $T^{\prime}$-ribbon, $\left(P^{\prime}, r^{\prime}\right)$. It is clear from our choice of ribbon tiles and glues that $\left(P^{\prime}, r^{\prime}\right)$ must consist of an infinite sequence of motifs. Hence we can construct an infinite $(T, d)$-directed zipper by replacing each motif with the tile $t$ of which it is a variant.

Theorem 4.2 proves the undecidability of existence of an infinite ribbon. This settles the open problem [17], stating the following: "Problem 4.1. Given a tiling system $T$, is there an infinite $T$-snake within the infinite grid $G=\mathbb{Z} \times \mathbb{Z}$ ?"

In the terminology of [17], a tiling system $T$ is exactly as defined in section 2 , a finite set of tiles, i.e., of squares with colored edges that cannot be rotated and with infinitely many copies of each tile available. The grid $G$ is the integer grid of positions in the plane. An infinite $T$-snake is a sequence of tiles on the plane in which successive tiles are adjacent along an edge and touching edges have the same color; i.e., infinite $T$-snakes are (possibly self-crossing) 2-way infinite ribbons where identical tiles must be present at the crossing sites. In general [17], [34], an infinite snake problem asks, given a tiling system $T$, and some portion $P$ of the plane, whether there is an infinite $T$-snake that lies entirely within $P$. Reference [17] proves that, given $T$ and a strip of width $k \in N$, the existence of an infinite $T$-snake that lies entirely within the strip is decidable. Given a tile system $T$, and a specific tile $t_{0} \in T$, the problem of whether there exists an infinite $T$-snake that contains $t_{0}$ is proved undecidable in [17] 
using methods in [16] (the case of a 1-way infinite snake starting at $t_{0}$ was proven undecidable in [15]). If the special "seed" tile is not specified, like in Problem 4.1, [17] conjectures the problem undecidable but states that "[...] it seems that this would be difficult to prove. We have not been able to adjust the proof techniques of other undecidability results for this purpose."

Theorem 4.2 , by showing the undecidability of existence of infinite non-selfcrossing snakes, proves Problem 4.1 undecidable.

5. Undecidability of self-assembly of arbitrarily large supertiles. Let us return to the discussion of self-assembly. Supertiles are constructed by an incremental process starting from a single tile and proceeding by addition of single tiles that "stick" to the hitherto built structure. The problem we are addressing is whether or not, given a tile system, an infinite supertile can self-assemble with tiles from that system. To formalize our notions, we have the following definition.

Definition 5.1. A shape is a function $f: I \longrightarrow \mathbb{Z}^{2}$ such that $I$ is a set of consecutive natural numbers, $0 \in I$, and the following condition holds. For all $i \in I$, if $i>0$, then there exists $j \in I$ such that $j<i$ and $f(i)$ and $f(j)$ are adjacent.

A shape thus describes a connected region of the plane. The size of a shape $f$ is the cardinality of its range, i.e., the number of positions it contains, regardless of how many times they are visited. If we fill in each position of a shape with tiles from a given tile system $T$, we obtain the notion of a $T$-supertile.

DEFINITION 5.2. For all tile systems $T$, a T-supertile is a pair $(f, g)$, where $f$ is a shape and $g:$ range $(f) \longrightarrow T$ is a function such that the following condition holds. For all $i \in \operatorname{dom}(f)$, if $i>0$, then there exists $j \in \operatorname{dom}(f)$ such that $j<i$ and $f(i)$ abuts $f(j)$ on the north (east, south, west) and $g(f(i))$ sticks on the north (east, south, west) to $g(f(j))$.

The size of a $T$-supertile $(f, g)$ is the size of its corresponding shape $f$. Two $T$-supertiles $(f, g)$ and $\left(f^{\prime}, g^{\prime}\right)$ are equivalent iff there exists $i, j \in \mathbb{Z}$ such that for all $(x, y) \in \mathbb{Z}^{2},(x, y) \in \operatorname{range}(f)$ iff $(x+i, y+j) \in \operatorname{range}\left(f^{\prime}\right)$ and for all $(x, y) \in \operatorname{range}(f)$, $g(x, y)=g^{\prime}(x+i, y+j)$. That is, the $T$-supertile $\left(f^{\prime}, g^{\prime}\right)$ is equivalent to the $T$-supertile $(f, g)$ iff $\left(f^{\prime}, g^{\prime}\right)$ can be obtained by translating $(f, g)$ on the plane.

Note that the definitions of shape and supertile may differ in other papers. The ideas are similar, but the details may not be the same. The problem stated at the beginning of this section is settled by the following result.

THEOREM 5.1. The following sets are undecidable:

$S_{1}=\{T \mid T$ is a tile system and there exists an infinite $T$-supertile $\}$;

$S_{2}=\{T \mid T$ is a tile system and there exists infinitely many nonequivalent finite T-supertiles\}.

Proof. It is easily shown that sets $S_{1}$ and $S_{2}$ are identical to the set $\{T \mid T$ is a tile system and there exists an infinite $T$-ribbon $\}$. Hence Theorem 5.1 follows from Theorem 4.2.

6. Conclusion. In this paper we prove the undecidability of the "unseeded version" of the problem of distinguishing tile systems that allow infinite ribbons to selfassemble from those that do not. The proof includes the construction of a special set of directed tiles with the so-called strong plane-filling property, whereby any directed zipper (a special kind of ribbon) is forced to be plane-filling and, moreover, such an infinite directed zipper always exists.

This result settles an open problem formerly known as the "unlimited infinite snake problem." 
We also prove the undecidability of the "unseeded version" of the problem of distinguishing tile systems that allow the self-assembly of infinite supertiles from those that do not.

We introduce a "motif" construction that allows one tile system to simulate another by using geometry to represent glues. This construction may be useful in other contexts.

Acknowledgments. We thank David Harel for pointing out that the "infinite ribbon problem" had been open since 1992 under the name "unlimited infinite snake problem," and Dale Myers and Yael Etzion-Petruschka for clarifications of [17]. We thank Qi Cheng, Ashish Goel, Ming-Deh Huang, Pablo Moisset de Espanes, and Paul Rothemund for discussion and comments.

\section{REFERENCES}

[1] H. Abelson, D. Allen, D. Coore, C. Hanson, G. Homsy, T. Knight, R. Nagpal, E. Rauch, G. Sussman, And R. Weiss, Amorphous computing, Comm. ACM, 43 (2000), pp. 74-82.

[2] L. Adleman, Molecular computation of solutions to combinatorial problems, Science, 266 (1994), pp. 1021-1024.

[3] L. Adleman, Towards a Mathematical Theory of Self-Assembly, Technical report 00-722, Department of Computer Science, University of Southern California, Los Angeles, CA, 2000.

[4] L. Adleman, Q. Cheng, A. Goel, And M. Huang, Running time and program size for self-assembled squares, in Proceedings of the ACM Symposium on Theory of Computing (STOC), 2001, pp. 740-748.

[5] L. Adleman, Q. Cheng, A. Goel, M. Huang, D. Kempe, P. Moisset de Espanes, and P. Rothemund, Combinatorial optimization problems in self-assembly, in Proceedings of the ACM Symposium on Theory of Computing (STOC), 2002, pp. 23-32.

[6] L. Adleman, Q. Cheng, A. Goel, M. Huang, and H. Wasserman, Linear self-assemblies: Equilibria, entropy, and convergence rates, in Proceedings of the Sixth International Conference on Difference Equations Augsburg, Germany 2001: New Progress in Difference Equations, B. Aulbach, S. Elaydi, and G. Ladas, eds., CRC Press, Boca Raton, FL, 2004, pp. 51-60.

[7] L. Adleman, J. Kari, L. Kari, And D. Reishus, On the decidability of self-assembly of infinite ribbons, in Proceedings of the IEEE Symposium on Foundations of Computer Science (FOCS), 2002, pp. 530-537.

[8] R. Barish, P. Rothemund, and E. Winfree, Two computational primitives for algorithmic self-assembly: Copying and counting, NanoLetters, 5 (2005), pp. 2586-2592.

[9] J. Bath, S. Green, and A. Turberfield, A free-running DNA motor powered by a nicking enzyme, Angew. Chem. Int. Edn., 44 (2005), pp. 4358-4361.

[10] Y. Benenson, T. Paz-Elizur, R. Adar, E. Keinan, Z. Livneh, and E. Shapiro, Programmable and autonomous computing machines made of biomolecules, Nature, 414 (2001), pp. 430-434.

[11] R. Berger, The undecidability of the domino problem, Mem. Amer. Math. Soc. 66 (1966), pp. 1-72.

[12] H. Chen, A. Goel, C. Luhrs, and E. Winfree, Self-assembling tile systems that heal from small fragments, in Preliminary Proceedings of DNA Computing 13, M. Garzon and H. Yan, eds., 2007, pp. 30-46.

[13] M. Cook, P. Rothemund, And E. Winfree, Self-assembled circuit patterns, in Proceedings of DNA Computing 9, Lecture Notes in Comput. Sci. 2943, J. Chen and J. Reif, eds., Springer, Berlin, 2004, pp. 91-107.

[14] M. Dubois, B. Demé, T. Gulik-Krzywlcki, J.-C. Dedieu, C. Vautrin, S. Désert, E. Perez, AND T. Zemb, Self-assembly of regular hollow icosahedra in salt-free catanionic solutions, Nature, 411 (2001), pp. 672-675.

[15] H. Ebbinghaus, Domino threads and complexity, in Computation Theory and Logic, Lecture Notes in Comput. Sci. 270, E. Borger, ed., Springer, Berlin, 1987, pp. 131-142.

[16] Y. Etzion, On the Solvability of Domino Snake Problems, M.Sc. thesis, Department of Applied Mathematics and Computer Science, The Weizmann Institute of Science, Rehovot, Israel, 1991.

Copyright $@$ by SIAM. Unauthorized reproduction of this article is prohibited. 
[17] Y. Etzion-Petruschka, D. Harel, and D. Myers, On the solvability of domino snake problems, Theoretic. Comput. Sci., 131 (1994), pp. 243-269.

[18] K. Fujibayashi, R. Hariadi, S. Park, E. Winfree, and S. Murata, Toward reliable algorithmic self-assembly of DNA tiles: A fixed-width cellular automaton pattern, NanoLetters, 8 (2008), pp. 1791-1797.

[19] A. Goel And P. Moisset de Espanes, Towards minimum size self-assembled counters, in Proceedings of DNA Computing 13, M. Garzon and H. Yan, eds., Lecture Notes in Comput. Sci. 4848, Springer, Berlin, 2008, pp. 46-53.

[20] M. Gomez-Lopez, J. Preece, And J. Stoddart, The art and science of self-assembling molecular machines, Nanotechnology, 7 (1996), pp. 183-192.

[21] R. Goodman, I. Schaap, C. Tardin, C. Erben, R. Berry, C. Schmidt, and A. Turberfield, Rapid chiral assembly of rigid DNA building blocks for molecular nanofabrication, Science, 310 (2005), pp. 1661-1665.

[22] D. Gracias, J. Tien, T. Breen, C. Hsu, and G. Whitesides, Forming electrical networks in three dimensions by self-assembly, Science, 289 (2000), pp. 1170-1173.

[23] S. Green, L. Lubrich, AND A. Turberfield, DNA hairpins: Fuel for autonomous DNA devices, Biophys. J., 91 (2006), pp. 2966-2975.

[24] D. HilberT, Über die stetige Abbildung einer Linie auf ein Flächtenstück, Math. Ann., 38 (1891), pp. 459-460.

[25] M. KAO AND R. SCHwelleR, Reducing tile complexity for self-assembly through temperature programming, in Proceedings of the ACM-SIAM Symposium on Discrete Algorithms (SODA), 2006, pp. 571-580.

[26] J. KARI, Reversibility of $2 D$ cellular automata is undecidable, Phys. D, 45 (1990), pp. 379-385.

[27] J. KARI, Reversibility and surjectivity problems of cellular automata, J. Comput. System Sci., 48 (1994), pp. 149-182.

[28] D. KöNIG, Über eine Schlussweise aus dem Endlichen ins Unendliche, Acta Litt. Sci. Szeged, 3 (1927), pp. 121-130.

[29] M. Lagoudakis and T. LaBean, 2D DNA self-assembly for satisfiability, in DNA Based Computers V, DIMACS Ser. Discrete Math. Theoret. Comput. Sci. 54, E. Winfree and D. Gifford, eds., AMS, Providence, RI, 2000, pp. 141-154.

[30] T. Liedl And F. Simmel, Switching the conformation of a DNA molecule with a chemical oscillator, NanoLetters, 5 (2005), pp. 1894-1898.

[31] G. Lopinski, D. WAyner, AND R. Wolkow, Self-directed growth of molecular nano-structures on silicon, Nature, 406 (2000), pp. 48-51.

[32] C. Mao, T. LaBean, J. Reif, and N. Seeman, Logical computation using algorithmic selfassembly of DNA triple-crossover molecules, Nature, 407 (2000), pp. 493-496.

[33] B. Muller, A. Reuter, F. Simmel, ANd D. Lamb, Single-pair FRET characterization of DNA tweezers, NanoLetters, 6 (2006), pp. 2814-2820.

[34] D. Myers, Decidability of the tiling connectivity problem, abstract 79T-E42, Notices Amer. Math. Soc., 195 (1979), A-441.

[35] R. Plass, J. Last, N. Bartelt, and G. KellogG, Nanostructures: Self-assembled domain patterns, Nature, 412 (2001), p. 875.

[36] J. REIF, Local parallel biomolecular computation, in DNA Based Computers III, DIMACS Ser. Discrete. Math. Theoret. Comput. Sci. 48, H. Rubin and D. Wood, eds., AMS, Providence, RI, 1999, pp. 217-254.

[37] R. Robinson, Undecidability and nonperiodicity for tilings of the plane, Invent. Math., 12 (1971), pp. 177-209.

[38] P. Rothemund, Using lateral capillary forces to compute by self-assembly, Proc. Natl. Acad. Sci. USA, 97 (2000), pp. 984-989.

[39] P. Rothemund, Theory and Experiments in Algorithmic Self-Assembly, Ph.D. thesis, University of Southern California, Los Angeles, CA, 2001.

[40] P. Rothemund, Folding DNA to create nanoscale shapes and patterns, Nature, 440 (2006), pp. 297-302.

[41] P. Rothemund, N. Papadakis, and E. Winfree, Algorithmic self-assembly of DNA Sierpinski triangles, PLoS Biol., 2 (2004), e424.

[42] P. Rothemund and E. Winfree, The program-size complexity of self-assembled squares, in Proceedings of the ACM Symposium on Theory of Computing (STOC), 2001, pp. 459-468.

[43] J. Schön, H. MENG, AND Z. BAO, Self-assembled monolayer organic field-effect transistors, Nature, 413 (2000), pp. 713-715.

[44] W. Sherman and N. Seeman, A precisely controlled DNA biped walking device, NanoLetters, 4 (2004), pp. 1203-1207.

[45] W. Shin, J. Quispe, And G. Joyce, A 1.7-kilobase single-stranded DNA that folds into a 
nanoscale octahedron, Nature, 427 (2004), pp. 618-621.

[46] D. Soloveichik And E. Winfree, Complexity of self-assembled shapes, SIAM J. Comput., 36 (2007), pp. 1544-1569.

[47] Y. Tian, Y. He, Y. Peng, And C. MaO, A DNA enzyme that walks processively and autonomously along a one-dimensional track, Angew. Chem. Int. Edn., 44 (2005), pp. 43554358.

[48] H. WANG, Proving theorems by pattern recognition, Bell System Tech. J., 40 (1961), pp. 1-42.

[49] G. Whitesides, J. Mathias, and C. Seto, Molecular self-assembly and nanochemistry: A chemical strategy for the synthesis of nanostructures, Science, 254 (1991), pp. 1312-1319.

[50] E. Winfree, Algorithmic Self-Assembly of DNA, Ph.D. thesis, California Institute of Technology, Pasadena, CA, 1998.

[51] E. Winfree, F. Liu, L. Wenzler, and N. Seeman, Design and self-assembly of twodimensional DNA crystals, Nature, 394 (1998), pp. 539-544.

[52] E. Winfree, X. YAng, And N. Seeman, Universal computation via self-assembly of DNA: Some theory and experiments, in DNA Based Computers II, DIMACS Ser. Discrete Math. Theoret. Comput. Sci. 44, L. Landweber and E. Baum, eds., AMS, Providence, RI, 1999, pp. 191-213.

[53] B. Yurke, A. Turberfield, A. Mills, Jr., F. Simmel, and J. Neumann, A DNA-fuelled molecular machine made of DNA, Nature, 406 (2000), pp. 605-608.

Copyright $@$ by SIAM. Unauthorized reproduction of this article is prohibited. 